\title{
Phenotype plasticity as enabler of melanoma progression and therapy resistance
}

DOI:

10.1038/s41568-019-0154-4

\section{Document Version}

Accepted author manuscript

Link to publication record in Manchester Research Explorer

\section{Citation for published version (APA):}

Arozarena, l., \& Wellbrock, C. (2019). Phenotype plasticity as enabler of melanoma progression and therapy resistance. Nature Reviews Cancer, 19(7), 377-391. https://doi.org/10.1038/s41568-019-0154-4

\section{Published in:}

Nature Reviews Cancer

\section{Citing this paper}

Please note that where the full-text provided on Manchester Research Explorer is the Author Accepted Manuscript or Proof version this may differ from the final Published version. If citing, it is advised that you check and use the publisher's definitive version.

\section{General rights}

Copyright and moral rights for the publications made accessible in the Research Explorer are retained by the authors and/or other copyright owners and it is a condition of accessing publications that users recognise and abide by the legal requirements associated with these rights.

\section{Takedown policy}

If you believe that this document breaches copyright please refer to the University of Manchester's Takedown Procedures [http://man.ac.uk/04Y6Bo] or contact uml.scholarlycommunications@manchester.ac.uk providing relevant details, so we can investigate your claim.

\section{OPEN ACCESS}




\title{
Phenotype plasticity as enabler of melanoma progression and therapy resistance
}

\author{
Imanol Arozarena ${ }^{1^{*}}$ and Claudia Wellbrock ${ }^{2 *}$ \\ ${ }^{1}$ Navarrabiomed, Complejo Hospitalario de Navarra (CHN), Universidad Pública de Navarra (UPNA), \\ Instituto de Investigación Sanitaria de Navarra (IdiSNA), Pamplona, Spain ${ }^{2}$ Division of Cancer \\ Sciences, Faculty of Biology, Medicine and Health, University of Manchester, Manchester, UK; \\ *email: Claudia.wellbrock@manchester.ac.uk; Imanol.arozarena.martinicorena@navarra.es
}

\begin{abstract}
Malignant melanoma is notorious for its inter- and intra-tumour heterogeneity based on transcriptionally distinct melanoma cell phenotypes. It is thought that these distinct phenotypes are plastic in nature, and that their transcriptional reprogramming enables heterogeneous tumours to undergo different stages of melanoma progression and to adjust to drug exposure during treatment. Recent advances in genomic technologies and the rapidly expanding availability of large gene expression data sets allowed for a refined definition of the gene signatures that characterise these phenotypes, and have revealed that phenotype plasticity plays a major role in the resistance to targeted therapy as well as immunotherapy. In this review we will discuss the definition of melanoma phenotypes through particular transcriptional states and reveal the prognostic relevance of the related gene expression signatures. We will review how the establishment of phenotypes is controlled, and which role phenotype plasticity plays in melanoma development and therapy. Because phenotype plasticity in melanoma bears great resemblance to epithelial mesenchymal transition (EMT), lessons learned from melanoma will also benefit our understanding of other cancer types.
\end{abstract}




\section{[H1] Introduction}

Cutaneous malignant melanoma carries one of the highest mutational burdens ${ }^{1}$. Nevertheless, prominent driver mutations in for instance the BRAF or NRAS gene are established early during melanoma development and already detectable in benign nevi ${ }^{2}$. Furthermore, analyses of cohorts of matched primary and metastatic melanomas have neither identified driver mutations that are restricted to metastases, nor a conclusive hierarchic mutation pattern associated with metastatic progression ${ }^{3-7}$. However, melanoma progression to an invasive state is linked to an increase in copy number alterations ${ }^{8}$. This suggests that changes in gene expression and hence transcriptional programmes play a role in melanoma progression.

Gene expression analyses of cultured melanoma cells have established the existence of two predominant transcriptional programmes. These programmes are expressed in distinct cell populations defined as either of 'proliferative' or 'invasive' phenotype [G] ${ }^{9-13}$, a terminology that has been widely adopted also when describing cell populations within the tumour tissue. Over the years the gene expression signatures related to these two phenotypes have been confirmed and expanded (Table 1). Crucially, the proliferative and invasive phenotypes are not defined by particular genetic lesions, and changes in transcriptional activity can reprogram a phenotype. Moreover, the transcriptomes of the invasive and proliferative phenotype are governed by distinct transcriptional master regulators ${ }^{14-16}$. Consequently, depending on the balance between the expression and/or activity of transcriptional master regulators, cells are capable of adaptive phenotype plasticity or 'phenotype switching'. Importantly, the occurrence of phenotype plasticity, reminiscent of the epithelial mesenchymal transition (EMT) [G] and mesenchymal-epithelial transition (MET) [G] of epithelial cancer cells, has been observed in vivo ${ }^{17}$.

Considering that transcriptional changes are induced through signalling pathways activated by extracellular factors, the tumour microenvironment (TME) plays a major role in adaptive phenotype plasticity. Different non-cancer cells in the TME as well as metabolic conditions such as oxygen and nutrient supply can modify phenotypes and drive them from proliferative to invasive and vice versa ${ }^{9,11-13}$. These microenvironmental effects can explain the inter- as well as intra-tumour phenotype heterogeneity frequently observed in melanoma. Indeed, histological analyses of melanoma biopsies revealed distinct expression of the melanoma transcriptional master regulator microphthalmia-associated transcription factor (MITF), a marker of the proliferative phenotype, and the tyrosine-protein kinase receptor AXL, a marker for the invasive phenotype ${ }^{18}$. This heterogeneity can also be detected at RNA level as populations of invasive cells with a MITF ${ }^{\text {high }}$ and proliferative cells with an $\mathrm{AXL}^{\text {high }}$ transcriptional programme ${ }^{19}$. Accordingly, these two markers are commonly used to identify phenotype populations described as MITFF ${ }^{\text {high }}-\mathrm{AXL}^{\text {low }}$ and MITF ${ }^{\text {low }}-\mathrm{AXL}^{\text {high }}$.

More recently the application of single cell analysis and advanced genomic technologies has led to a more refined definition of phenotype related transcriptional states ${ }^{19-21}$ and has underlined their importance for melanoma, particularly in the context of therapy. Emerging evidence suggests that phenotype plasticity is at the core of the development of resistance to targeted therapy but also to immunotherapy ${ }^{20,22}$, making it a major obstacle for the cure of melanoma. 
In this review we will discuss how melanoma phenotypes are defined through gene expression signatures, examine their prognostic relevance, and describe how the establishment of phenotypes is controlled. Overall, we will discuss the role of phenotype plasticity in melanoma progression and therapy response, and how key processes in phenotype plasticity could be therapeutic targets used in novel treatment options.

\section{[H1] Phenotype specific gene signatures}

As early as 2000 it was suggested that particular gene expression patterns could be used to describe phenotypic behaviour of cultured melanoma cells, and help with a molecular classification of melanoma ${ }^{23}$. In particular, a gene expression 'cluster' was identified linked to the melanocyte markers endothelin receptor type $\mathrm{B}$ (EDNRB) and melan-A (MLANA) distinct from a different transcriptome related to $W N T 5 A$ and $A X L^{23}$ (Table 1). Cell lines expressing a WNT5A-AXL-transcriptome displayed increased invasion in vitro ${ }^{23}$. Later on, further gene expression signatures were identified and led to the definition of the 'invasive' phenotype marked by TGF $\beta$ and the 'proliferative' phenotype marked by the expression of SOX10 and MITF ${ }^{14}$. Further refinement determined distinct gene expression of WNT5A and $A X L$ in the invasive phenotype, and reinforced MITF as marker of the proliferative phenotype $^{16}$ (Table 1). Of note, MITF is the master transcription regulator of the melanocyte-lineage [G], and apart from controlling melanoma cell survival and proliferation, it stimulates differentiation and pigmentation [G] through genes such as EDNRB and $M L A N A^{24}$, which are markers of gene expression signatures linked to the proliferative phenotype ${ }^{23,25}$. Moving on from cell lines, later studies included data sets from melanoma biopsies and applied RNAseq, which allowed significantly expanding the size of the individual gene sets (Table 1). This study validated and expanded previously defined signatures, and identified a MITF-SOX10 governed proliferative signature that contained $68-99 \%$ and an AP1TEAD governed invasive signature that contained $65-69 \%$ of previously defined gene signatures ${ }^{16,18}$. Of note, the signatures do not correlate with the presence of any known melanoma driver mutation such as $B R A F^{\mathrm{V} 600 \mathrm{E}}$ or $N R A S^{\mathrm{Q} 61 \mathrm{~L}}$, which suggests transcriptional reprogramming occurs, leading to gene expression programs that are disconnected from driver mutations ${ }^{14,15}$.

Analyses of datasets from biopsies also enabled the identification of a gene expression signature that distinguishes melanoma from other cancers, and as such is up-regulated specifically in melanoma ${ }^{26}$ (Table 1). Mapping these genes onto cell lines of invasive or proliferative phenotypes revealed a proliferative signature enriched in genes that promote melanocyte differentiation, which are MITF, its target genes and its up-stream regulators SOX10 and PAX3. Intriguingly, the analysis did not identify a single overlap with the 643 genes defining the invasive signature ${ }^{15}$. Because the study aimed to identify a signature containing 'melanoma specific' genes ${ }^{26}$, this suggests that invasive signature genes are not specific for melanoma but might be applicable to more cancer types.

In general, a fairly clear picture of the gene expression landscape in melanoma cells is emerging. The invasive and proliferative phenotype gene signatures describe part of a series of melanoma cell states that relate to different phases of melanocyte lineage development, ranging from neural crest stem cells to differentiated, pigmented melanocytes ${ }^{20,21}$ (Table 1). 
Nevertheless, when these signatures are 'mapped' onto tumours, expression of genes reflecting the tumour micro-environment, in particular genes specific to immune cells and associated with a lymphocyte infiltrate can be identified ${ }^{15,19,27}$. Moreover, while bulk sequencing of tumours can identify dominance of either a proliferative or invasive phenotype signature, single cell analyses revealed that both phenotype signatures can coexist and contribute to intra-tumour heterogeneity ${ }^{19,28}$. Such heterogeneity can be seen macroscopically in melanomas that consist of radial and vertical growth components ${ }^{29,30}$, and can explain gene expression signature variations found in individual metastases derived from the same primary tumour ${ }^{31}$.

\section{[H1] Phenotype plasticity and cooperativity}

The intra-tumour phenotype heterogeneity found in melanomas can be a direct result of the adaptive plasticity of melanoma transcriptomes, which enables the fluent conversion from one phenotype into another in response to external cues. Support for the concept of adaptive phenotype plasticity as a bona fide biological phenomenon comes from single-cell analyses of melanoma biopsies, which detected populations of cells with invasive or proliferative phenotype signatures, but also detected individual cells with signatures representative of what can be considered transition states [G] ${ }^{19,28}$.

\section{[H2] Phenotype plasticity and the phenotype-switch model.}

Phenotype plasticity in vivo has been corroborated by the observation that melanoma cell lines formed tumours in mice that ultimately displayed heterogeneity for MITF expression, regardless of whether the cell lines had a mainly invasive (MITF negative) or proliferative (MITF positive) phenotype in vitro ${ }^{17}$. This study laid the ground for the so called 'phenotypeswitch model', in which adaptive switching between different phenotypes in response to the TME is thought to drive melanoma progression (Figure 1a).

The phenotype-switch model captures features of progressed melanomas, which are not covered by other models involving cancer stem cells and clonal expansion (Box 1). In addition, various microenvironment factors that have been linked to melanoma progression can stimulate a phenotype switch. For instance extracellular ligands such as TGF $\beta^{14,32,33}$, WNT5 $A^{34-36}$ and TNF $\alpha^{13,37,38}$ (Table 2) induce a de-differentiated invasive phenotype. MITF seems to act as gatekeeper for the transition to an invasive phenotype, because only when MITF is suppressed, cells can transit from a proliferative to an invasive phenotype ${ }^{39}$. On the other hand, although the proliferative phenotype requires transcriptional activity of MITF, expression of MITF in invasive cells does not necessarily induce a proliferative phenotype, because it can result in suppression of melanoma cell proliferation and xenograft growth ${ }^{39,40}$. It appears that the transition from an invasive to a proliferative phenotype requires additional changes, such as epigenetic modifications, in order for MITF to drive transcription of proliferation and differentiation genes. Indeed, the chromatin landscape differs between fully established phenotypes; open chromatin at active enhancers in the proliferative phenotype overlaps with active enhancers in melanocytes and open chromatin at active enhancers in the invasive phenotype overlaps with active enhancers in skin fibroblasts ${ }^{15}$. In addition, regions of active and repressed chromatin are correlated with regions of decreased and increased methylation, respectively ${ }^{15}$, and in invasive phenotype cells the MITF 
promoter as well as the promoters of its target genes are hyper-methylated ${ }^{41}$. Consequently, ectopic MITF expression cannot stimulate expression of MITF target genes. Thus, the need for epigenetic modifications might explain why MITF overexpression alone cannot induce a phenotype switch in cell lines with a fully established invasive phenotype.

The question remains why the switch from a proliferative phenotype to an invasive phenotype can be experimentally achieved, while induction of the reverse switch seems more difficult (Table 2). Possibly cellular changes (in adhesion receptors, MMPs, etc) leading to invasive behaviour as measured by common in vitro assays can be induced fast, before the full establishment of a stable slow-cycling state corresponding to cells expressing the invasive phenotype signature. This would suggest that an invasive phenotype as determined by common in vitro assays does not necessarily overlap with the invasive phenotype as defined by gene signatures (Box2) and that the forced switch from a 'proliferative signature' to an 'invasive signature' phenotype might actually be more difficult. Indeed, SOx9 overexpression induces increased invasion in vitro, but this is accompanied by only a partial enrichment of only $10 \%$ of invasive signature genes ${ }^{42}$. Another example is seen with the neurotrophin receptor CD271, which is expressed in neural crest stem cells ${ }^{43}$, and although controversial $^{44-46}$, has been identified as marker of melanoma initiating cells ${ }^{47}$. CD271 overexpression in melanoma cells can induce transcription linked to an altered matrixadhesion phenotype within $24 \mathrm{~h}$, but at this time there is no major overlap with the invasive gene signature ${ }^{48}$. Finally, transcription regulators or epigenetic factors such as the EMT regulator $\mathrm{ZEB} 1^{49}$, as well as $\mathrm{HOXA1}^{50}$ or $\mathrm{BMI}^{51}$ can enhance in vitro invasion and increase expression of genes contained within the invasive signature, but do not induce a slow-cycling phenotype and their effects on proliferation are inconclusive (Box 2). Thus, although all these factors enhance invasive properties, they are not sufficient to consolidate a slowcycling phenotype. This suggests that for global reprogramming underlying plasticity between stable slow-cycling and invasive, and stable proliferative and differentiated states to occur, cells need to integrate various signals over a prolonged period of time; only this will enable a cell to establish an epigenetic state compatible with a stable phenotype (Figure 1b). Once a stable phenotype is established, a cell appears to be 'locked' in a transcriptional state supported by the respective epigenetic landscape. Such a scenario is in line with findings made in cells undergoing EMT, where transition states exist and plasticity is observed, but the epithelial or fully established mesenchymal states are less plastic ${ }^{52}$.

The importance of phenotype plasticity for melanoma progression has been elegantly demonstrated with an inducible CD271 system in mice ${ }^{48}$. Prolonged CD271 over-expression induces an invasive phenotype and reduces tumour growth in mice, but this can be recovered after receptor expression is switched off ${ }^{48}$. Most importantly, the reversible CD271 expression that allows for proliferation to recover is essential for efficient metastasis, because when CD271 overexpression is not switched off, metastasis does not occur. Similarly, melanoma cells of the invasive phenotype can only form metastasis in zebrafish if they undergo an EDN-3 induced switch to a proliferative gene programme (Table 1) once they arrive at the metastatic site ${ }^{12}$.

In order for a cell to disseminate it has to adopt invasive properties, but the findings described above suggest that its overall transcriptional state can stay transient allowing for microenvironment induced adaptive plasticity to occur. This plasticity promotes progression, 
because as seen with CD271 a fully established invasive phenotype alone is insufficient to cause metastatic disease, and a reversible switch to a proliferative phenotype is required for metastasis to develop. Reversibility is key to melanoma progression driven by phenotype plasticity, but whether one direction is favoured over the other, will also depend on the respective microenvironment, in which for instance one state is energetically more stable than the other (Figure 1b). Considering the impact of phenotype plasticity and metastatic growth on disease outcome, it will be crucial to identify which transition represents the bottleneck for the development of metastasis.

\section{[H2] Communication between phenotypes leads to cooperativity.}

The 'phenotype-switch model' postulates that during certain steps of the metastatic process we should encounter only invasive cells (Figure 1a). However, the phenotype heterogeneity found not only in individual metastases ${ }^{19}$, but also in circulating melanoma cells ${ }^{53,54}$ challenges the simplicity of this model. It seems likely that in a heterogeneous situation communication between cells of different phenotypes occurs. This can for example result in cooperative actions that overall further the metastatic process whilst maintaining heterogeneity (Figure 1c). In epithelial cancers there is growing evidence that cooperativity can occur and contribute to collective migration, efficient dissemination, pre-metastatic niche conditioning and the initiation of metastases ${ }^{55}$. Notably, also melanoma cells cooperate, and the communication between cells of the invasive phenotype with cells of the proliferative phenotype can increase the overall efficiency of invasion in vivo ${ }^{56}$. In this regard, heterogeneous MITF expression has also been detected in circulating melanoma cell clusters $^{53}$. Cells in circulation are challenged by cell death induced by detachment (anoikis), and MITF $^{\text {low }}$ invasive phenotype melanoma cells expressing the AP1 transcription factor subunit FOSL1 are resistant to anoikis ${ }^{57}$. Thus MITF ${ }^{\text {high }}$ cells, otherwise prone to anoikis, might receive a survival benefit from attachment to MITF $^{\text {low }}$ cells within circulating clusters. This would explain why proliferative phenotype genes are present in a 19-gene signature that specifies circulating melanoma cells in patients ${ }^{58}$.

Overall, although phenotype plasticity occurs, it cannot be dismissed that phenotype heterogeneity contributes to adaptation in cellular function beyond defined phenotypes.

\section{[H1] Biological and prognostic relevance}

Particular functions such as proliferation or invasion have been assigned to cells with distinct phenotype signatures, and this is most clearly represented in the phenotype switch model (Fig. 1a). However, several findings suggest that there is more to cells with an invasive phenotype signature than simply being invasive. Furthermore, the invasive phenotype is frequently referred to as the 'metastatic' and 'more aggressive' phenotype. Nevertheless, while independent approaches to isolate melanoma-classifiers with prognostic relevance identified gene expression signatures that overlap with the invasive and proliferative phenotype signatures (Table 1), these studies consistently found that it is the proliferative phenotype signature that correlates with poor survival. 


\section{[H2] Phenotype specific functions and melanoma development}

The definition of the invasive and proliferative phenotypes is based on distinct cell behaviour that has originally been assessed in particular in vitro and in vivo assays (Box 2), but in the context of melanoma development phenotype function appears to be less clear-cut.

The gene expression signature of the classic invasive phenotype, is void of any MITF transcripts $^{14,59}$, yet depending on context MITF can also stimulate invasion ${ }^{60-62}$. Moreover, MITF significantly up-regulates $27 \%$ invasive signature genes ${ }^{63}$, many of them encode extracellular matrix (ECM) proteins and matrix remodelers. This makes sense, considering that MITF expression is induced in neural crest-derivatives [G] when the melanocyte lineage evolves as highly motile cell population ${ }^{64}$. In fact, even adult melanocytes, which are defined by ample MITF expression, are highly motile. This trait is thought to underlie the formation of so called nevus cell aggregates, small deposits of melanocytes found in lymph nodes of patients and therefore named 'benign metastases' ${ }^{65,66}$. Presumably, when melanocytes encounter relevant external cues they are able to migrate and invade without the need to transform. Thus, the ability to disseminate might be an inherent trait of melanocytic cells and not an acquired feature of melanoma progression ${ }^{8}$. Following on from this, invasive phenotype cells may be defined by an increase in in vitro invasive capacities, but there seem to be other properties linked to this de-differentiated phenotype such as slow-cycling or dormancy, altered adhesion signalling and anoikis resistance in addition to drug resistance; these attributes are of major relevance to progression and metastasis ${ }^{67}$ as well as therapy ${ }^{20,21,68}$ and we still do not know enough about them.

The proliferative phenotype has probably maintained most of the traits of a melanocyte, in which both the MAP-kinase pathway and MITF drive proliferation ${ }^{24,69,70}$. In patients, metastases have the highest mitotic index of all melanoma stages ${ }^{71}$, emphasising the importance of proliferation. Indeed, induction of the proliferative programme at the metastatic site is a prerequisite for metastasis formation in fish $^{12}$, and MITF expression is required for metastatic out-growth in mice ${ }^{72}$. To fully comprehend the role of the proliferative phenotype and MITF expression in melanoma, we need to better understand their involvement in instigating proliferation in invasive phenotype or dormant cells and their contribution to survival at secondary sites.

\section{[H2] Prognostic value of melanoma phenotype signatures.}

The quest for identifying melanoma-classifying gene expression signatures is driven by the need for appropriate predictors of melanoma. One of the first attempts identified genes related to cell cycle progression and proliferation as significantly up-regulated in primary melanomas of patients who developed metastasis within the next 4 years following diagnosis $^{73}$ (Table 1). Remarkably similar correlations of poor patient survival with expression of proliferation and/or cell cycle genes were seen with several other melanoma cohorts including the TCGA panel of primary and metastatic melanomas ${ }^{27,71,74,75}$ (Table 1). In line with this, the mitotic index of melanomas appears to be the strongest predictor of poor outcome $^{71}$. On the other hand, a gene expression signature related to 'immune response' is correlated with better survival $\left.\right|^{27,71,74,75}$. 
MITF and its target genes regulating the cell cycle including CDK2 or BRCA1, as well as target genes regulating pigmentation are expressed in tumour biopsies of the poor survival patient groups, which is in line with classic proliferative gene signature ${ }^{71,74,75}$ (Table 1). The expression of pigmentation genes is in line with the fact that expression of melanocyte differentiation genes per se is also correlated with poor survival ${ }^{26,76}$. Although at first glance this might appear contradictory, the processes of proliferation and differentiation in melanocytic cells are not mutually exclusive, and this is because MITF has a dual role in controlling genes involved in proliferation and differentiation, as mentioned above. In fact, cell division of pigmented i.e., differentiated melanocytes occurs and has been monitored by live imaging ${ }^{77}$.

Another interesting observation is that although best survival correlates with the immune response group, this signature displays low expression of MITF and its target genes ${ }^{74}$, suggesting a possible phenotype specific interplay with the immune microenvironment. Indeed, single cell RNAseq of human melanoma biopsies revealed that T-cell infiltration is correlated with complement factors expressed by cancer-associated fibroblasts (CAFs) and that CAF-rich tumours express an $\mathrm{AXL}^{\text {high }}$ signature, whereas expression of a MITF $F^{\text {high }}$ programme in melanoma cells is negatively correlated with CAF abundance, and hence T-cell infiltration ${ }^{19}$. Thus apart from the cellular functions assigned to distinct phenotypes, the phenotype-induced TEM affects melanoma progression, and the interplay of melanoma cells with the TEM might contribute to the poor prognosis seen in patients with tumours of proliferative phenotype signature.

\section{[H1] Multiple factors control phenotypes}

Whereas certain traits of melanoma cells are inherent to the melanocytic lineage, locally distinct factors in the TME affect melanoma phenotypes and contribute to phenotype heterogeneity (Figure 2). Likewise, melanoma cells of distinct phenotypes can shape the melanoma microenvironment, because the effect of intercellular communication based on cell-cell interactions and secreted factors is phenotype-dependent .

\section{[H2] Resident-cells in the tumour microenvironment control melanoma phenotypes.}

Within a tumour there is a constant flow of communications between cancer cells and other cell types in the microenvironment; these communications are reciprocal and contribute to the establishment of certain phenotype. Major contributors to these interactions are $\mathrm{CAFs}^{78}$ (Figure 2a). Indeed, co-culture of melanoma cells with CAFs leads to induction of an invasive MITF $^{\text {low }}-A X L^{\text {high }}$ phenotype in melanoma cells ${ }^{79}$, and this phenotype is enriched in melanomas with high CAF abundance ${ }^{19}$. Ageing CAFs become more likely to induce an invasive AXL ${ }^{\text {high }}$ phenotype in melanoma cells $\mathrm{s}^{80}$, and this induction is further facilitated by reduced secretion of ECM components and remodelers such as hyaluronan and proteoglycan link protein 1 (HPLN1), which lowers matrix stiffness to favour invasive behaviour ${ }^{81}$. But the interplay between the ECM, CAFs and melanoma cells is complex. While in tumours with low relative amounts of CAFs, collagen abundance and therefore ECM stiffness triggers differentiation and a MITF ${ }^{\text {high }}$ phenotype, the abundance of CAFs can overcome this effect, because CAFderived TGF $\beta$ stimulates dedifferentiation ${ }^{76}$ (Figure 2a). Mechanistically, ECM stiffness drives MITF expression through a YAP-PAX3 complex, but the presence of TGF $\beta$ stimulates 
recruitment of YAP to TEAD, which suppresses MITF expression. Thereby, YAP acts as crucial rheostat between a MITF and TEAD driven transcriptional state ${ }^{76}$.

The relevance of the ECM in the TME can be seen in patient survival data, where worst prognosis is seen in patients with melanoma in which collagen expression is high, and which also express MITF target genes ${ }^{76}$. What has not been considered yet is the factor of age, because not only do ageing fibroblasts contribute to a change in EXM composition, but also the structure of matrix proteins such as collagen changes through accumulation of UV damage over time ${ }^{82}$. Nevertheless, also acute UV irradiation affects melanoma progression, and this is not only through causing DNA damage in melanocytes, but also through an inflammation-mediated switch to a de-differentiated state. This switch produces a motile phenotype that is prone to metastatic spread along blood vessels (angiotropism) ${ }^{37}$ (Figure $2 \mathrm{~b})$. Curiously, the melanoma cells detected along these blood vessels display pigmentation and expression of the differentiation markers TRP1 and S100. This exemplifies the transient nature of the switch, and suggests that once the cells are located along the vessels and local inflammation has subsided, the cells can switch back to a state of differentiation and continue to migrate.

Central to the inflammation-mediated phenotype plasticity is the pro-inflammatory cytokine TNF $\alpha$ (Table 2), which is produced by neutrophils, activated T-cells and myeloid cells. TNF $\alpha$ suppresses MITF and induces the expression of JUN, a subunit of the transcription factor AP1 (see Fig. 1b), leading to a pro-inflammatory MITF ${ }^{\text {low }}$-JUN ${ }^{\text {high }}$ state ${ }^{38}$. However, MITF binds to and blocks the JUN promoter, and in cells expressing high MITF levels, TNF $\alpha$ is not able to induce JUN and thus inefficient to induce a switch to the JUN ${ }^{\text {high }}$ phenotype. Moreover, when the MAP-kinase pathway upstream of JUN is inhibited, TNF $\alpha$ induces MITF expression through TF65/RELA, which binds to the MITF promoter ${ }^{83}$. It appears therefore that not all melanoma cells respond to TNF $\alpha$ equally. Rather, cells need to be primed for the pro-inflammatory action of TNF $\alpha$, and this priming is related to the ability of TNF $\alpha$ to upregulate JUN expression. Once established, the MITF ${ }^{\text {low }}{ }_{-} U N^{\text {high }}$ state displays inflammatory hyper-responsiveness, which in mice promotes a myeloid cell enriched $\mathrm{TME}^{38}$ (Figure 2b). In a mouse model for adoptive cell transfer therapy (ACT), TNF $\alpha$ induced phenotype plasticity enables melanoma cells to escape cytotoxic T-cells, which contributes to therapy resistance ${ }^{13}$. A similar escape is seen when melanoma cells are co-cultured with NK cells, which induce a mesenchymal phenotype in melanoma cells ${ }^{84}$. These observations could suggest that switching to a MITF ${ }^{\text {low }}$ phenotype is correlated with escaping an immuneresponse. However, while exposure to TNF $\alpha$ resulted in the escape from melanocyte differentiation antigen (MDA) specific T-cells, the recognition of melanoma cells by T-cells specific for non-melanocytic antigens appeared to be unaffected or even increased ${ }^{13}$. In line with this, downregulation of MITF in human melanoma cells in vitro results in increased cytokine release and immune cell attraction ${ }^{85,86}$. Furthermore, in patients with melanoma, an immune response signature correlated with increased lymphocyte infiltration and better survival was found to be related to a MITF ${ }^{\text {low }}$ state and absence of proliferative markers ${ }^{71,74}$. Thus, the MITF ${ }^{\text {low }}$ state might be more immunogenic or is connected to a microenvironment that effectively recruits immune cells, as it was seen in CAF-rich $A \mathrm{XL}^{\text {high }}$-signature melanomas with higher T-cell infiltration ${ }^{19}$. 


\section{[H2] Control through physiological conditions in the tumour microenvironment.}

The presence of different resident cells and other factors within the TME also influences the availability of oxygen or nutrients (Figure 2a), which in turn contributes to the adaptation of melanoma phenotypes to optimise survival and growth. At sites of non-functional blood vessels, low levels of oxygen (hypoxia) directly affect cells of the TME including melanoma cells. In particular, hypoxia promotes a de-differentiated phenotype in melanoma cells, which has lower oxygen consumption and is therefore more likely to survive ${ }^{87}$. A further driving force for a switch to a dedifferentiated phenotype could be that this phenotype is capable of vasculogenic mimicry [G] and as such can contribute to neovascularisation ${ }^{88-90}$. Mechanistically, the switch is linked to MITF suppression mediated by the hypoxia-induced transcription factor HIF-1 $\alpha$, which stimulates expression of the transcriptional repressor BHE40, which in turn binds and suppresses the MITF promoter and hence promotes dedifferentiation 9,59,91,92 (Table 2). Under normoxic conditions, MITF induces the expression of PRGC1, a transcriptional co-activator that promotes mitochondrial energy metabolism and reactive oxygen species (ROS) detoxification ${ }^{87}$. However, in hypoxic cells when MITF is suppressed, cells are more glycolytic and ROS levels are elevated, and hence, these dedifferentiated cells are exposed to oxidative stress. Importantly, HIF-1 $\alpha$ in its role in oxygen-availability related stress signalling has also been linked to counteracting ROS production ${ }^{93}$. HIF-1 $\alpha$ could therefore protect cells of the MITF ${ }^{\text {low }}$ dedifferentiated phenotype from elevated ROS levels. Intriguingly, ROS levels are also increased when aged fibroblast induce a switch to a dedifferentiated phenotype ${ }^{80}$, but a potential role for HIF-1 $\alpha$ in this scenario is not known.

Because the rate of oxygen consumption is directly linked to intracellular energy production from nutrients, nutrient availability is also a crucial factor in regulating melanoma cell phenotypes. For instance glucose restriction leads to reduction in glycolytic flux and ROS accumulation, which via ATF4 induces transcriptional repression of MITF and a switch to a MITF ${ }^{\text {low }}$-AXL ${ }^{\text {high }}$ phenotype ${ }^{11}$ that is inefficient at oxidative phosphorylation ${ }^{11,87}$. Restriction of the amino acid glutamine also leads to the establishment of a MITF ${ }^{\text {low }}-$ AXL $^{\text {high }}$ phenotype, whereby the repression of MITF expression and repression of pro-invasive activities are mediated by transcriptional and translational events ${ }^{10}$. Overall, it seems that oxygen and nutrient limitation leads to adopting a slow-cycling phenotype, which is more likely to sustain the harsh metabolic conditions in the TME. Of note, melanoma cells expressing a 'nutrient deprived cells' signature also express high levels of the fatty acid transporter $\mathrm{CD} 36^{20}$, suggesting that they are primed to increased fatty acid up-take. CD36 labels slowcycling, undifferentiated carcinoma cells, and in animal models CD36 regulates the metastatic capacity of several cancer types, including melanoma ${ }^{94}$. Thus, fatty acids might fuel melanoma progression, but so far nothing is known about the role of CD36 and fatty acid metabolism in slow-cycling melanoma cells.

\section{[H1] Phenotype plasticity during therapy}

MAP-kinase inhibitors (MAPKis) effectively block proliferation in BRAF mutated MITF ${ }^{\text {high }}$ melanomas, but most patients eventually progress ${ }^{95,96}$. While therapy resistance based on acquired mutations is partially to blame for this ${ }^{97}$ mounting evidence points to a parallel 
process, in which the plasticity of melanoma transcriptomes allows for adaptation of cells to the presence of drug and drug resistance ${ }^{98}$. Immune check-point inhibitors can produce durable responses, in $\sim 16-40 \%$ of patients with a follow-up up to 10 years ${ }^{99-101}$, yet up to $30 \%$ of patients who responded for a period of time relapse on treatment ${ }^{95,102}$. Also here, phenotype plasticity plays a role, because individual phenotypes differ in their immunogenicity and in their interaction with the immune-microenvironment ${ }^{103,104}$.

\section{[H2] Phenotype plasticity drives resistance to targeted therapy.}

It is now well established that melanomas adapt when they are challenged by MAPKi and studies in cultured cells revealed that during this adaptation transcriptionally distinct cell populations emerge over time with an initial phase of populations showing increased melanocytic differentiation (MITF ${ }^{\text {high }}$ ), followed by continuous dedifferentiation and the appearance of cells with a slow-cycling neural crest-like state (NGFR $\left.{ }^{\text {high }}\right)^{105-107}$. A similar pattern of consecutive transcriptional states is seen in patient biopsies and in patientderived xenograft (PDX) models ${ }^{20,21}$ (Table 1). Crucially, in patients with melanoma undergoing treatment with BRAF inhibitor (BRAFi) and MEK inhibitor (MEKi) the respective transcriptional states in the tumour can co-exist at the initial drug-response phase, although there is clear inter-patient variability with regard to predominant states (Figure $3 a)^{20}$.

In the initial response-phase up to $78 \%$ of melanomas of patients on treatment with MAPKi display an increase in MITF $^{\text {high }}$ cells ${ }^{20,108}$ (Figure 3a), and this can result in a drug-tolerant state, because MITF mediated survival-signalling can counteract MAPKi induced cell death ${ }^{108,109}$. In parallel, cell populations expressing an invasive as well as a neural-crest stem cell-like signature increase in number and often co-emerge within the same tumour ${ }^{20}$ (Figure 3a). These cell states correlate with the enrichment of a repertoire of receptor tyrosine kinases (RTKs) such as NGFR ${ }^{20}$, PFGFR ${ }^{110}$, IGF1R $^{111}, \mathrm{EGFR}^{112}, \mathrm{AXL}^{19,113,114}$ or ROR2 ${ }^{92}$, and this is paralleled by the lack of MITF and its up-stream regulators SOX10 or PAX3 ${ }^{15,112}$ (Table 1). Mechanistically it is thought that the aforementioned RTKs provide survival signals that are independent of MAPK signalling; hence these RTK expressing MITF ${ }^{\text {low }}$ melanoma cells are resistant to $\mathrm{MAPKi}^{113,114}$. This might explain, why at the relapsed stage $70 \%$ of melanomas show increased expression of the most prominent resistance marker $\mathrm{AXL}^{115}$, (Figure 3a), resulting in an overall trend towards an AXL-driven transcriptional programme in these resistant tumours ${ }^{19}$. Accordingly, MITF expression is reduced in $\sim 50 \%$ of relapsed melanomas ${ }^{108}$. Nevertheless, $\sim 23 \%$ of melanomas relapse with up-regulated MITF expression ${ }^{108}$ (Figure 3a), which apart from transcriptional plasticity can also be based on MITF gene amplifications, an event correlated with disease progression ${ }^{116,117}$.

Gene expression analysis can identify changes in individual transcriptional states in tumours on treatment, but in vitro single cell analyses was required to demonstrate that these states are based on transcriptional reprogramming rather than on selection. These analyses identified the existence of individual cells that co-express increased levels of several resistance markers such as EGFR, AXL or tumour necrosis factor receptor superfamily member 16 (TNFR16, also known as NGFR) ${ }^{21,105-107}$. These cells appear to be primed for resistance and BRAFi treatment leads to further up-regulation of these markers and a transient, reversible resistance. In line with what is observed in patients, these changes are paralleled by loss of SOX10 and increase in TEAD and AP-1 expression ${ }^{106}$. 
As mentioned above, exposure to MAPKi can enrich for de-differentiated slow-cycling drugtolerant melanoma cell populations, and these populations display chromatin modifications and up-regulation of histone demethylases ${ }^{21,68,118,119}$. Dedifferentiation appears to be a frequent response to MAPKis, which would allow these slow-cycling melanoma cells to persist until acquisition of mutations leads to established resistance ${ }^{120}$. In vitro studies suggest that the de-differentiated state can be transient, but it has also been shown that prolonged BRAFi incubation can stabilize this state ${ }^{21,106,107}$, presumably through epigenetic reprogramming as seen in tumours from patients with acquired resistance ${ }^{121}$. A stabilisation of MAPKi -induced resistance is seen in drug-addicted melanoma cells, in which removal of drug results in cell death, presumably because the cells are unable to adjust quickly enough to switch back to alternate survival signalling ${ }^{122}$. Predicting reversibility of MAPKi resistance is crucial when considering therapy strategies involving drug holidays and drug-rechallenge.

\section{[H2] Inter-cell communication contributes to resistance.}

Despite the obvious complexity provided by tumour heterogeneity, we know surprisingly little about how communication between cells of different phenotypes influences the responses to targeted therapy. In fact, the MAPK-pathway regulates the expression of numerous secreted proteins and MAPKis profoundly alter the composition of these secretomes ${ }^{123,124}$ which in turn stimulate novel cell-cell interactions (Figure 3a). Within a heterogeneous tumour this can lead to the nurturing of stably resistant clones ${ }^{123}$, but can also provide vital growth signals to slow-cycling phenotypes ${ }^{124}$ (Figure $3 b$ ). Monitoring phenotype populations over time of treatment suggests that there are significant differences between BRAFi and MEKi combination therapy and BRAFi monotherapy. In particular, the former accelerates enrichment of the slow-cycling $A X L^{\text {high }}$ phenotype, but the latter delays enrichment for $\mathrm{AXL}^{\text {high }}$ cells ${ }^{19,115}$, possibly due to inter-phenotype communications that maintain phenotype heterogeneity ${ }^{124}$. A factor that is involved in such inter-phenotype communications is EDN1, which is secreted by MITF ${ }^{\text {high }}$ cells in response to BRAFi and stimulates proliferation of $\mathrm{AXL}^{\text {high }}$ cells ${ }^{124}$ (Figure $3 a$ and $\mathrm{b}$ ).

\section{[H2] Phenotype plasticity affects immunotherapy.}

Several genetic events have been linked to innate resistance to immunotherapy ${ }^{102}$, but tumours from patients who do not respond to anti-programmed cell death 1 (PD1) therapy are also enriched in a mesenchymal-transition related transcriptome termed innate anti-PD1 resistance signature (IPRES) $)^{22}$. The IPRES displays similarities to MAPKi-resistance signatures including expression of $A X L, R O R 2$ and WNT5A, which hints at a shared concept of nongenomic resistance. In line with this, in a mouse model for ACT, where resistance is based on lack of T-cell recognition due to absence of MDAs, transcriptional states of progressive dedifferentiation are observed ${ }^{13,21}$. Ultimately, transcriptional changes underlying IPRES may be stabilised through epigenetic changes ${ }^{15,41}$. Indeed, the use of de-methylation or histone deacetylase (HDAC) inhibitors has been demonstrated to improve immunotherapy by reactivating expression of immune-related genes and tumour antigens ${ }^{125}$. Thus, overall similar to the way melanoma cells escape from targeted therapy, melanoma cells seem to be able to escape immunotherapy through transcriptional plasticity with a trend towards a mesenchymal phenotype and dedifferentiation. 
The link between melanoma dedifferentiation and therapy escape is not always as clear. For example, desmoplastic melanoma typically presents as a fibrotic tumour of invasive cells displaying reduced pigmentation, increased expression of ECM and neurotrophic factors, and frequent amplifications of RTK genes ${ }^{126,127}$, which are characteristics of the dedifferentiated mesenchymal phenotype. However, this melanoma variant has a very high mutational burden, which makes it highly immunogenic resulting in a favourable response to immune checkpoint blockade ${ }^{128}$. Thus, in context of an increased mutational burden and immune response it seems that transcriptional states linked to loss of MDA expression can be counterbalanced by the mutational burden resulting in neo-antigens.

Furthermore, the genetic background of the melanoma may also affect its interaction with the immune system. This is not only seen with different oncogenic drivers in various mouse models ${ }^{13,103,104}$, but also represented in patients. For instance, in tumour biopsies from patients with melanoma, $\beta$-catenin mutations and increased canonical WNT signalling, which is linked to melanocyte differentiation, was found to correlate with T-cell exclusion ${ }^{103}$. This finding was corroborated in $\mathrm{Braf}^{\mathrm{V} 600 \mathrm{E}} \mathrm{Pten}^{-/}$mice in which mutant $\beta$-catenin expressing melanomas displayed increased MDA expression, yet T-cells were excluded due to the lack of a subset of dendritic cells ${ }^{103}$. Moreover, these differentiated melanomas did not respond effectively to immune checkpoint therapy. In contrast, in an $\mathrm{H}-\mathrm{Ras}^{G 12 \mathrm{~V}} / \mathrm{p} 16^{-/}$driven mouse model, de-pigmented melanomas that have switched to the mesenchymal phenotype recruit T-cells, but stimulate apoptosis in these through recruitment of polymorphonuclear myeloid-derived suppressor cells (PMN-MDSC) ${ }^{104}$. Overall these highly de-differentiated melanomas are resistant to immune checkpoint therapy and ACT.

Responses to immunotherapy can be durable, but with an increasing number of patients receiving this treatment, it is becoming clearer that acquired resistance poses a problem. The advantage of immunotherapy is the capacity of immune cells to memorise. However, numerous distinct populations of immune cells and complex cell-cell communications are involved in an effective immune response, which makes it difficult to pinpoint one or two simple mechanisms responsible for resistance ${ }^{102}$. Research into immunotherapy resistance is still in its early days, but it is conceivable that apart from the inherent immune-background of the host and the mutational burden and driver mutations of the tumour, phenotype plasticity will also affect the outcome of immunotherapy.

\section{[H2] Therapeutic opportunities based on targeting phenotypes.}

A major take home message from our understanding of the concept of phenotype plasticity is that responses to MAPKi in melanoma can be reversible. This means that if at the time of relapse tumours have not accumulated genetic changes that drive resistance, taking advantage of this reversibility is a realistic opportunity. Indeed, success of the concept of drug holidays has been experimentally demonstrated ${ }^{129}$, and emerging evidence suggests that re-challenging patients with MAPKi after relapse can be successful ${ }^{130,131}$.

Moreover, based on our knowledge of the melanoma phenotype dynamics occurring in response to MAPKi therapy (Figure 3a), strategies can be envisaged that will monitor markers of the individual transcriptional states during treatment, followed by adjusting treatment with appropriate drug combinations. For instance, in the first weeks of MAPKi treatment, a MITF ${ }^{\text {high }}$ state is seen in various patients ${ }^{20,108,120,132}$, which can be overcome by 
blocking MITF up-regulation using nelfinavir ${ }^{108}$, or targeting MITF mediated survival signals and metabolic adaptation ${ }^{109,133,134}$. Monitoring the slow-cycling $\mathrm{AXL}^{\text {high }}$ population on treatment has confirmed its enrichment at the time of relapse ${ }^{19,124}$. Approaches to target this phenotype can take advantage of its ROS ${ }^{\text {high }}$ state using ROS or lipid peroxide-mediated ferroptosis [G] inducing drugs ${ }^{21,87}$. Besides, although originally assigned to the proliferative signature $^{15}$, reanalysis identified the nuclear retinoid acid receptor RXRG as driver of the slow-cycling neural crest stem cell (NCSC)-like population, and co-targeting RXR delays the onset of MAPKi resistance ${ }^{20}$. More directly, the AXL-specific antibody AXL-107-MMAE cooperates with MAPKi to eradicate this resistant population ${ }^{115}$. Considering all these specific approaches, certainly an optimal approach would target both the MITF ${ }^{\text {high }}$ and $\mathrm{AXL}^{\text {high }}$ populations simultaneously, which would require a target that displays phenotypespecific expression yet exhibits equal functionality in both phenotypes. This condition is fulfilled with the EDN1 receptors EDNRB and EDNRA, which are expressed in MITF ${ }^{\text {high }}$ and $\mathrm{AXL}^{\text {high }}$ cells, respectively (Figure $3 \mathrm{~b}$ ). Consequently, blocking EDNR signalling not only profoundly extends the time of BRAFi response, but also effectively suppresses the enrichment of $A X^{\text {high }}$ inhibitor-resistant cells ${ }^{124}$.

Today treatment options often include both MAPKi and immunotherapy. Combinations of these approaches are currently explored and apart from challenging toxicities responses are very varied ${ }^{135}$. In this context the interplay of melanoma phenotypes plays an important role; for instance up-regulation of a MITF ${ }^{\text {high }}$ phenotype on BRAFi therapy is correlated with improved T-cell recognition ${ }^{136}$, whereas WNT5A reprograms local dendritic cells to induce immune tolerance ${ }^{137}$. It will be important to establish whether phenotype plasticity and reversibility plays a role, as this might inform on how one treatment regime can set the scene for the other treatment, and whether acquired resistant tumours from one treatment will still respond to the other treatment.

Great focus has been placed on revealing phenotype specific changes related to resistance to therapy in order to improve responses. However, there are other aspects to phenotype plasticity that could be targeted. For instance, stable slow-cycling cells are not sufficient to cause metastatic disease, because this requires a switch to proliferation. However, we know very little about how proliferation is initiated in a slow-cycling, possibly dormant cell. These cells must rely on phenotype specific survival mechanisms, but while the mechanism by which MITF mediates survival signalling in MITF ${ }^{\text {high }}$ cells is well-explored, it is not established what provides survival signals in the slow-cycling phenotype. Moreover, in a heterogeneous tumour different phenotypes and their transition states co-exist and we have just begun to touch on the complexity of interactions that occur as a consequence of phenotype heterogeneity. Considering that such communications promote cooperativity -also during therapy- isolating the players involved in these interactions should help identifying potent therapeutic targets.

\section{[H1] Conclusions and Perspectives}

Phenotype plasticity is central to melanoma initiation, progression, and resistance to therapy. Since the first definition of two distinct melanoma phenotypes it is has emerged 
that identifiable melanoma cell state signatures relate to different development phases of the melanocyte lineage. Hence phenotypes rather than being only invasive or proliferative are more complex and describe a melanocyte differentiation gradient. Accordingly, identifying invasiveness or proliferation as function in melanoma cells appears to not necessarily equal a phenotype defined by gene signature. Furthermore, while MITF induces differentiation, studies analysing the dedifferentiated and invasive phenotype so far only focus on the act of MITF suppression in the process of generating this phenotype. Apart from this, we are only beginning to understand the dynamic changes in chromatin states that are underlying phenotype plasticity.

In summary, there is need to revisit the original 'invasive' and 'proliferative' phenotype definition and to further investigate the factors that take over in the absence of MITF, possibly AP1, TEADs and SMADs. We have to improve our knowledge about the dynamics of the epigenetic landscapes and their related gene regulatory networks that drive transition states or stabilise phenotypes. Most importantly, we need to better understand how phenotypes affect TEM composition, and vice versa how the TEM controls phenotypes as this has major implications for therapeutic approaches. Finally, for prognostic purposes an agreement on a signature-taxonomy to define 'master-signatures' and their association with parameters of melanoma pathology is urgently required. The use of such master-signatures can improve diagnosis and monitoring therapy response. Moreover, their functional analysis particularly in the context of the TEM can lead to the identification of targetable master regulators and novel means to target plasticity to improve current treatment strategies. 


\section{Box 1 I A role for melanoma stem cells or clonal evolution in melanoma development?}

Numerous studies have demonstrated that primary and secondary melanomas display high similarities in genetic make-up as well as gene expression patterns ${ }^{4-6,8,73}$. This fact strongly argues against a role for clonal evolution and expansion of the fittest clone after competitive selection as a major driver of melanoma progression ${ }^{138}$. Nevertheless, an alternative concept that is discussed involves cancer stem cells (CSCs), which are transformed cells with normal stem cell characteristics that are thought to drive unlimited tumour growth in a hierarchical fashion ${ }^{138}$. This concept is accepted for various cancer types, but the relevance of CSCs for melanoma progression has been a matter of debate for some time. While melanoma cell sub-populations that display characteristics of stemness, such as extensive self-renewal or single cell tumour initiation have been frequently identified, inconsistencies in verifying established markers of CSCs have undermined efforts to confirm their existence ${ }^{44,139,140}$. The concept of CSCs in melanoma has also been challenged based on its postulation that only rare sub-populations of cells within a tumour have the capacity to initiate and sustain tumour growth, because it was demonstrated that in $28 \%$ of cases single melanoma cells directly isolated from patients could initiate tumours and were capable of indefinite growth $^{46}$. In addition, the aforementioned study showed that the phenotypic heterogeneity observed in initial xenografts was maintained after injection of individual markerfractionated cell populations. Together this suggests not only that tumour-initiating cells are rather common in melanoma, but the findings also support the concept of non-hierarchical plasticity. It has been suggested that such plasticity could lead to the transient existence of slow-cycling cells with stem cell properties ${ }^{141}$, which has been named 'dynamic stemness'. Such a concept is supported by the existence of melanoma cell sub-populations that possess neural crest features and are further de-differentiated than the classic invasive phenotype (NCSC, Table 1), but most importantly show dynamic behaviour ${ }^{20,21}$. Thus, though perceived as difficult to define, melanoma CSCs could in fact be plastic neural crest stem cell-like cells that contribute to melanoma growth and progression as well as to therapy resistance. 


\section{Box 2 I Assessing the function of the invasive and proliferative phenotypes}

Bittner and co-workers ${ }^{23}$ were the first to link a particular gene signature to invasive behaviour, which they measured as matrigel-invasion towards foetal calf serum (FCS); a standard assay still used to define this phenotype. Thereby, invasion depends on FCS responsive receptors, which are highly expressed in invasive signature cells (e.g. EGFR, PDGFR, AXL). Thus, proliferative signature cells may not respond to FCS as chemo-attractant, yet they exhibit invasive activities. Indeed, cell lines defined as proliferative can display higher basal matrigel-invasion than invasive lines ${ }^{15}$, and they can use other chemoattractants such as EDN1/EDN3 ${ }^{142}$. Furthermore, the proliferative phenotype is considered 'non-metastatic'. However, depending on the immune-background in mice, proliferative phenotype cells can be highly metastatic ${ }^{94}$, even more metastatic than invasive signature cells (C.W. and I.A. personal communication), suggesting that the immunogenicity of individual phenotypes in mice affects their 'metastatic' potential.

Correlating the proliferative signature to proliferation in vitro is inconclusive ${ }^{15-17,26,51}$, but the first study evaluating phenotypes in vivo found that proliferative phenotype cell lines produced rapidly growing tumours within 10 days, whereas tumour growth for invasive signature cell lines occurred 30 days later and was slow ${ }^{17}$. This is intriguing as the efficiency to grow a tumour is considered a feature of tumorigenic potential, also linked to dedifferentiated tumour initiating cells, and indeed other studies find that only cell lines of invasive phenotype grow tumours, but the proliferative phenotype does not ${ }^{26,49,50,143}$. Furthermore, inducers of an invasive phenotype like BMI1, HOXA1 or ZEB1 should reduce proliferation, but while BMI1 has no effect ${ }^{51}$, HOXA1 actually accelerates tumour growth ${ }^{50}$ and depletion of ZEB1 results in no tumour growth at all ${ }^{49}$. Overall, there seems to be no consensus on how to confirm melanoma phenotypes with regard to 'proliferation', which poses a problem for interpreting a phenotype switch, particularly in vivo.

Based on their gene expression profiles, it is possible to assign particular functions to phenotypes. However, with a stark focus on invasive behaviour and the lack of any agreed standard for how and when to assess proliferative behaviour in vivo we might be missing important features of these phenotypes that have major importance for melanoma progression. 
Figure 1 I Phenotype plasticity during melanoma progression

a I In the 'phenotype-switch' model, the proliferative phenotype drives initial tumour growth and a switch to an invasive phenotype establishes cells able to invade and disseminate ${ }^{17}$. At the metastatic site a switch back to the proliferative phenotype must occur to promote growth. b I In this 'phenotype-plasticity' model a stable phenotype, linked to a low-energy state can convert into another stable phenotype, but conversion requires activation energy for the establishment of transition states. Stable melanoma phenotypes are defined by transcriptomes that relate to either differentiated melanocytes or slow-cycling cells with neural crest stem cell-signatures ${ }^{20,21}$; the transition states reflect sequential dedifferentiation and differentiation between the stable phenotypes. A threshold must be overcome to transit between stable states, but the energy level of the stable states and hence the threshold might differ for the individual phenotypes. Different transcriptional master regulators are active on either side of the threshold. MITF, downstream of for instance MCR1, EDNRB or BRAF/MAPK signalling, controls differentiation and proliferation ${ }^{24}$. AP1-factors (FRA1, JUN), which are up-regulated for instance by MAPK-pathway hyper-activation ${ }^{122}$ or possibly through TNF $\alpha^{38}$, suppress MITF and stimulate dedifferentiation. WNT5A and TGF $\beta$ activate TEADs and SMADs respectably, which together can drive further dedifferentiation and establish a slow-cycling phenotype ${ }^{144,145}$. Of note, this model assumes that MITF-expressing cells also possess invasive potential, possibly when pigmentation levels are ${ }^{\circ} w^{33}$. c I Reciprocal interaction between different phenotypes results in cooperativity and maintenance of heterogeneity during melanoma progression. Phenotype plasticity occurs, but is not strictly required at all times.

Figure 2 I Control of phenotypes by microenvironment factors

a I Cancer-associated fibroblasts (CAFs) are a source of secreted factors such as TGF $\beta$ or extracellular matrix (ECM) proteins ${ }^{78}$, all of which can induce an MITF ${ }^{\text {low }}-\mathrm{AXL}^{\text {high }}$ phenotype. The secretome of ageing fibroblasts, which become increasingly senescent, contains additional factors such as SFRP2, which contribute to MITF down-regulation and induction of a de-differentiated $\mathrm{AXL}^{\text {high }}$ phenotype ${ }^{80}$. Also melanoma cells can actively deposit $\mathrm{ECM}^{56}$, and in the absence of CAFs and TGF $\beta$, this ECM induces a MITF ${ }^{\text {high }}$-phenotype ${ }^{76}$. Hypoxia induces a HIF-1 $\alpha$-mediated up-regulation of ROR2 expression, and WNT5A through ROR2 further stabilises HIF-1 $\alpha$ and establishes a MITF ${ }^{\text {low }}$-AXL ${ }^{\text {high }}$ phenotype ${ }^{35,92}$. Glucose and glutamine restriction induce a MITF ${ }^{\text {low }}$-AXL ${ }^{\text {high }}$ phenotype by down-regulating MITF through ATF $4^{10,11}$. Importantly, the hypoxia, as well as glucose, or glutamine restriction induced switch leads to MAPKi therapy resistance ${ }^{10,92,118}$. b I Immune cells are the source of numerous secreted factors that control cellular-behaviour and have a major impact on melanoma cell phenotype plasticity. UV irradiation triggers the recruitment of neutrophils, which induce inflammation and through TNF $\alpha$ stimulate angiotropism, whereby extravascular migration of pigmented differentiated cells can be seen ${ }^{37}$. During inflammation, activated $\mathrm{T}$ lymphocytes or myeloid cells also produce TNF $\alpha$. Depending on the expression level of JUN, TNF $\alpha$ can induce a MITF ${ }^{\text {low }-J U N^{\text {high }}}$ that shows hyper-responsiveness to cytokines, which furthers a proinflammatory and myeloid cell-rich tumour microenvironment. In a mouse model for adoptive cell transfer (ACT) therapy, establishment of a MITF ${ }^{\text {low }}$-JUN ${ }^{\text {high }}$ state results in loss of melanoma differentiation antigen (MDA) expression and T lymphocyte recognition. 
Figure 3 I Phenotype plasticity and therapy response

a I MITF ${ }^{\text {high }}$ tumours are responsive to MAPKi therapy, because BRAF mediated proliferation and survival depends on $\mathrm{MITF}^{69}$. In the initial response phase, transcriptional reprogramming is induced, but different transcriptional states or phenotypes can co-exist resulting in heterogeneous tumours. Inter-patient variability exists with regard to the relative presence of phenotypes, resulting in overall MITF ${ }^{\text {high }}$ (pigmented) or $\mathrm{AXL}^{\text {high }}$ (invasive) or NGFR ${ }^{\text {high }}$ (NCSC) phenotype tumours ${ }^{20}$. In tumours, where MITF up-regulation dominates, this initiates MITF-mediated drug tolerance ${ }^{108,120}$. Further into the treatment, adaptive transcriptional reprogramming and cell-cell communications via the drug-induced secreted factors EDN $1^{124}$ is induced. Thereby, EDN1, secreted by MITF ${ }^{\text {high }}$ cells provides pro-proliferative signals to $\mathrm{AXL}^{\mathrm{high}}$ cells and enhances drug-tolerance. At the stage of acquired resistance the majority ( 70\%) of melanomas are enriched with the $\operatorname{AXL}^{\text {high }}$ phenotype ${ }^{19,114}$, but $\sim 23 \%$ of resistant tumours represent with increased MITF expression. $\mathbf{b}$ I The MAPK-pathway downstream of mutated BRAF regulates proliferation and survival in $\mathrm{MITF}^{\text {high }}$ cells, but it only regulates cellcycle progression in $A \mathrm{XL}^{\text {high }}$ cells; survival signals are provided through other signals ${ }^{124}$. Therefore a BRAFi will kill MITF high cells but only inhibit cell cycle progression in the already slow-cycling $A X L^{\text {high }}$ cells. MITF ${ }^{\text {high }}$ cells express EDRNB and $A X L^{\text {high }}$ cells express EDNRA, but the receptors do not signal effectively in the absence of ligand. BRAFi induces EDN1 secretion in MITF ${ }^{\text {high }}$ cells, and in a heterogeneous tumour, EDN1 allows AXL $^{\text {high }}$ cells to bypass BRAFi action through EDNRA, thereby promoting the outgrowth of an $\mathrm{AXL}^{\text {high }}$ population ${ }^{124}$. EDN1 also enables MITF ${ }^{\text {high }}$ cells to bypass the BRAFi insult via EDNRB, but the cells are still more vulnerable than $\mathrm{AXL}^{\text {high }}$ cells, presumably because the EDN1 mediated survival signals are weak. Blocking EDN1 signalling in combination with BRAFi effectively prolongs the BRAFi response and suppresses the enrichment of the $A X L^{\text {high }}$ population. 
Table1 I Melanoma gene expression Signatures

\begin{tabular}{|c|c|c|c|}
\hline Source & Signature & Identified markers & Reference \\
\hline \multicolumn{4}{|l|}{ Phenotype signatures } \\
\hline $\begin{array}{l}31 \text { samples } \\
\text { ( } 26 \text { cell lines, } 5 \text { biopsies) }\end{array}$ & $\begin{array}{l}\text { Cluster of } 19 \text { samples (contains } \\
\text { all biopsies), cell lines in this } \\
\text { cluster are less invasive }\end{array}$ & $\begin{array}{l}\text { outside cluster: WNT5A, AXL } \\
\text { in cluster: EDNRB, MART-1 /MLANA }\end{array}$ & 23 \\
\hline $\begin{array}{l}86 \text { cell lines ( } 3 \text { panels) } \\
\text { (metastatic, in situ) }\end{array}$ & $\begin{array}{l}\text { Motif } 1 \text { (invasive): } 51 \text { genes } \\
\text { Motif } 2 \text { (proliferative): } 54 \text { genes }\end{array}$ & $\begin{array}{l}\text { Motif 1: TGF } \beta \\
\text { Motif 2: MITF, SOX10 }\end{array}$ & 14 \\
\hline $\begin{array}{l}25 \text { metastases derived } \\
\text { cell lines }\end{array}$ & $\begin{array}{l}\text { Motif } 1 \text { (invasive): } 37 \text { genes } \\
\text { Motif } 2 \text { (proliferative): } 59 \text { genes }\end{array}$ & $\begin{array}{l}\text { Motif 1: HIF1 } \alpha, \text { TGF } \beta \\
\text { Motif 2: MITF, EDNRB }\end{array}$ & 25 \\
\hline 166 cell lines & $\begin{array}{l}\text { AXL-pos correlated: } 144 \text { genes } \\
\text { AXL-neg correlated: } 112 \text { genes }\end{array}$ & $\begin{array}{l}\text { AXL-pos: WNT5A, TGF } \beta \\
\text { AXL-neg: MITF, EDNRB }\end{array}$ & 18 \\
\hline $\begin{array}{l}218 \text { cell lines/short-term } \\
\text { cultures }\end{array}$ & $\begin{array}{l}\text { Invasive: } 45 \text { genes } \\
\text { Proliferative: } 52 \text { genes }\end{array}$ & $\begin{array}{l}\text { Invasive: WNT5A, AXL } \\
\text { Proliferative: MITF }\end{array}$ & 16 \\
\hline $\begin{array}{l}11 \text { short-term cultures } \\
\text { RNAseq biopsy datasets }\end{array}$ & $\begin{array}{l}\text { Invasive: } 642 \text { genes } \\
\text { Proliferative: } 770 \text { genes }\end{array}$ & $\begin{array}{l}\text { Invasive: TEAD, AP1 } \\
\text { Proliferative: MITF, SOX10, RXRG, CDH19 }\end{array}$ & 15 \\
\hline $\begin{array}{l}63 \text { melanoma cell lines } \\
\text { and biopsy datasets from } \\
8 \text { cancer types }\end{array}$ & $\begin{array}{l}\text { Up in melanoma: } 55 \text { genes } \\
\text { Cluster } 2 \text { (invasive): } 18 \text { genes } \\
\text { Cluster } 1 \text { (prolif.): } 37 \text { genes }\end{array}$ & $\begin{array}{l}\text { Cluster 2: GAS7, LEF1, RXRG, CDH19 } \\
\text { Cluster 1: MITF, SOX10, PAX3 }\end{array}$ & 26 \\
\hline \multicolumn{4}{|l|}{ Prognostic signatures } \\
\hline $\begin{array}{l}83 \text { primary melanomas } \\
58:>4 \text { years follow-up }\end{array}$ & $\begin{array}{l}254 \text { gene-classifier } \\
\text { 4Y no metastasis }(\mathrm{M}-) \text { : } 57 \text { genes } \\
\text { 4Y metastasis }(\mathrm{M}+): 197 \text { genes }\end{array}$ & $\begin{array}{l}\text { M- : EMX2 (MITF suppressor), CTNNBIP1 } \\
\text { (CTNNB inhibitor) } \\
\text { M+: PCNA, CDK1, BUB1, CENPA, CCNB2 }\end{array}$ & 73 \\
\hline $\begin{array}{l}44 \text { metastatic melanomas } \\
\text { follow-up: } 20 \text { months }\end{array}$ & $\begin{array}{l}266 \text { survival associated genes } \\
>1.5 \text { years survival } \\
<1.5 \text { years survival }\end{array}$ & $\begin{array}{l}>1.5 \text { years survival: ZAP70, LCK, CD3, } \\
\text { CXCL13 } \\
<1.5 \text { years survival: CDK2, PDE4D, ANLN }\end{array}$ & 71 \\
\hline $\begin{array}{l}57 \text { stage IV melanomas } \\
\text { follow-up: } 2 \text { years }\end{array}$ & $\begin{array}{l}\text { High-immune: } 100 \text { genes } \\
\text { Normal-like: } 77 \text { genes } \\
\text { Pigmentation: } 100 \text { genes } \\
\text { Proliferative: } 100 \text { genes }\end{array}$ & $\begin{array}{l}\text { High-immune: LCK, IFNGR1 } \\
\text { Normal-like: KRT10, KRT17 } \\
\text { Pigmentation: MITF, EDNRB } \\
\text { Proliferative: E2F1, BUB1, CCNA2 }\end{array}$ & 75 \\
\hline $\begin{array}{l}223 \text { primary melanomas } \\
\text { follow-up: } 10 \text { years }\end{array}$ & $\begin{array}{l}\text { Low-grade: } 177 \text { genes } \\
\text { high-immune } \& \text { normal-like } \\
\text { High-grade: } 200 \text { genes } \\
\text { proliferative/pigmentation }\end{array}$ & $\begin{array}{l}\text { Low-grade: MITF }{ }^{\text {low }}, \text { SOX } 10^{\text {low }}, \text { BRCA } 1^{\text {low }} \\
\text { High-grade: MITF } \\
\text { high } \\
\text { SOX } 10^{\text {high }}, \text { BRCA } 1^{\text {high }}\end{array}$ & 74 \\
\hline $\begin{array}{l}329 \text { primary } \& \\
\text { metastases } \\
\text { follow-up: } 10 \text { years }\end{array}$ & $\begin{array}{l}\text { Immune: } 111 \text { genes } \\
\text { MITF-low: } 166 \text { genes } \\
\text { Keratin: } 113 \text { genes }\end{array}$ & $\begin{array}{l}\text { Immune: CD8, NKG7, CD19 } \\
\text { MITF-low: CDH2, NRG1, BDNF } \\
\text { Keratin: KRT17, DCT, TYRP, SILV, CDH1 }\end{array}$ & 27 \\
\hline \multicolumn{4}{|l|}{ MAPKi therapy phenotypes } \\
\hline $\begin{array}{l}\text { In vitro melanocyte } \\
\text { differentiation model } \\
\text { mapped onto cell lines \& } \\
\text { biopsies }\end{array}$ & $\begin{array}{l}\text { Undifferentiated: } 224 \text { genes } \\
\text { Neural-crest like: } 197 \text { genes } \\
\text { Transitory: } 179 \text { genes } \\
\text { Melanocytic: } 187 \text { genes }\end{array}$ & 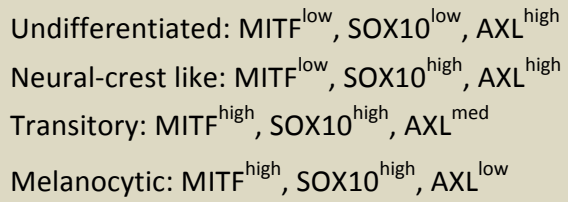 & 21 \\
\hline
\end{tabular}


PDX models, patient biopsies

BRAFi time course to resistance
Neuro (NCSC): 37 genes

Invasion: 49 genes

Hypometabolic (SMC): 27 genes

Pigmentation, MITF-targets: 124 genes
NCSC: MITF ${ }^{\text {low }}$, SOX10 $0^{\text {high }}$, NGFR $^{\text {high }}$ Invasion: MITF ${ }^{\text {low }}$, SOX10 $10^{\text {low }}$

SMC: MITF ${ }^{\text {med }}, \mathrm{CD} 6^{\text {med }}$

Pigmentation: MITF ${ }^{\text {high }}$, SOX10 $10^{\text {high }}$

Table 2 I Factors that induce phenotypes during phenotype switching

\begin{tabular}{|c|c|c|}
\hline Factor & $\begin{array}{l}\text { induced } \\
\text { phenotype }\end{array}$ & Effect in melanoma \\
\hline TGF $\beta$ & invasive & $\begin{array}{c}\text { Growth inhibition }^{14} \\
\text { Dedifferentiation, Motility }^{33} \\
\text { Invasion }^{32}\end{array}$ \\
\hline WNT5A & invasive & $\begin{array}{c}\text { Invasion }^{36} \\
\text { Suppression of differentiation }^{34} \\
\text { Metastasis }^{92} \\
\text { BRAF inhibitor resistance }\end{array}$ \\
\hline $\begin{array}{r}\text { TNF } \alpha / J U N / N F-\kappa B \\
\text { Inflammation }\end{array}$ & invasive & $\begin{array}{c}\text { Dedifferentiation }^{38} \\
\text { Angiotropism, Motility, Metastasis }^{37} \\
\text { BRAF inhibitor resistance }\end{array}$ \\
\hline CD271/NGFR & invasive & $\begin{array}{l}\text { Tumour growth inhibition } \\
\text { Metastasis }^{48}\end{array}$ \\
\hline ZEB1 & invasive & $\begin{array}{c}\text { EMT, Invasion }{ }^{49} \\
\text { BRAF inhibitor resistance }\end{array}$ \\
\hline HOXA1 & invasive & Tumour growth, Invasion, Metastasis ${ }^{50}$ \\
\hline BMI1 & invasive & Invasion, Metastasis ${ }^{51}$ \\
\hline Sox9 & invasive & Growth inhibition, Invasion, Metastasis ${ }^{42}$ \\
\hline $\begin{array}{l}\text { Hypoxia } \\
\text { HIF1 } \alpha\end{array}$ & invasive & $\begin{array}{c}\text { Invasion }^{9,59,91} \\
\text { BRAF inhibitor resistance }\end{array}$ \\
\hline $\begin{array}{l}\text { Nutrient starvation } \\
\text { (glucose, glutamine) }\end{array}$ & invasive & $\begin{array}{l}\text { Invasion }^{10,11} \\
\text { BRAF inhibitor resistance }\end{array}$ \\
\hline $\begin{array}{c}\mathrm{AXL} / \mathrm{NFKB} \\
\mathrm{AXL}\end{array}$ & invasive & BRAF inhibitor resistance ${ }^{113,114}$ \\
\hline FOSL1/FRA1, JUN & invasive & BRAF inhibitor resistance ${ }^{122,147,148}$ \\
\hline MITF & proliferative & $\begin{array}{l}\text { Target gene expression }^{63} \\
\text { Proliferation, Tumour growth }\end{array}$ \\
\hline EDN3 & proliferative & Differentiation, Proliferation ${ }^{12}$ \\
\hline
\end{tabular}




\section{References}

1 Alexandrov, L. B. et al. Signatures of mutational processes in human cancer. Nature 500, 415 421, doi:10.1038/nature12477 (2013).

2 Pollock, P. M. et al. High frequency of BRAF mutations in nevi. Nat Genet 33, 19-20, doi:10.1038/ng1054 (2003).

3 Ding, L. et al. Clonal architectures and driver mutations in metastatic melanomas. PLoS One 9, e111153, doi:10.1371/journal.pone.0111153 (2014).

4 Gartner, J. J. et al. Comparative exome sequencing of metastatic lesions provides insights into the mutational progression of melanoma. BMC Genomics 13, 505, doi:10.1186/1471-2164-13505 (2012).

5 Nikolaev, S. I. et al. Exome sequencing identifies recurrent somatic MAP2K1 and MAP2K2 mutations in melanoma. Nat Genet 44, 133-139, doi:10.1038/ng.1026 (2011).

6 Sanborn, J. Z. et al. Phylogenetic analyses of melanoma reveal complex patterns of metastatic dissemination. Proc Natl Acad Sci U S A 112, 10995-11000, doi:10.1073/pnas.1508074112 (2015).

7 Turajlic, S. et al. Whole genome sequencing of matched primary and metastatic acral melanomas. Genome Res 22, 196-207, doi:10.1101/gr.125591.111 (2012).

Shain, A. H. \& Bastian, B. C. From melanocytes to melanomas. Nat Rev Cancer 16, 345-358, doi:10.1038/nrc.2016.37 (2016).

9 Cheli, Y. et al. Hypoxia and MITF control metastatic behaviour in mouse and human melanoma cells. Oncogene 31, 2461-2470, doi:10.1038/onc.2011.425 (2012). Falletta, P. et al. Translation reprogramming is an evolutionarily conserved driver of phenotypic plasticity and therapeutic resistance in melanoma. Genes Dev 31, 18-33, doi:10.1101/gad.290940.116 (2017).

Ferguson, J., Smith, M., Zudaire, I., Wellbrock, C. \& Arozarena, I. Glucose availability controls ATF4-mediated MITF suppression to drive melanoma cell growth. Oncotarget 8, 32946-32959, doi:10.18632/oncotarget.16514 (2017).

12 Kim, I. S. et al. Microenvironment-derived factors driving metastatic plasticity in melanoma. Nat Commun 8, 14343, doi:10.1038/ncomms14343 (2017). Landsberg, J. et al. Melanomas resist T-cell therapy through inflammation-induced reversible dedifferentiation. Nature 490, 412-416, doi:10.1038/nature11538 (2012). Hoek, K. S. et al. Metastatic potential of melanomas defined by specific gene expression profiles with no BRAF signature. Pigment Cell Res 19, 290-302 (2006). regulators of the invasive cell state. Nat Commun 6, 6683, doi:10.1038/ncomms7683 (2015). Widmer, D. S. et al. Systematic classification of melanoma cells by phenotype-specific gene expression mapping. Pigment Cell Melanoma Res 25, 343-353, doi:10.1111/j.1755148X.2012.00986.x (2012). invasive states. Cancer research 68, 650-656, doi:10.1158/0008-5472.CAN-07-2491 (2008). Sensi, M. et al. Human cutaneous melanomas lacking MITF and melanocyte differentiation antigens express a functional Axl receptor kinase. J Invest Dermatol 131, 2448-2457, doi:10.1038/jid.2011.218 (2011).

Tirosh, I. et al. Dissecting the multicellular ecosystem of metastatic melanoma by single-cell RNA-seq. Science 352, 189-196, doi:10.1126/science.aad0501 (2016). Rambow, F. et al. Toward Minimal Residual Disease-Directed Therapy in Melanoma. Cell, doi:10.1016/j.cell.2018.06.025 (2018).

21 Tsoi, J. et al. Multi-stage Differentiation Defines Melanoma Subtypes with Differential Vulnerability to Drug-Induced Iron-Dependent Oxidative Stress. Cancer Cell 33, 890-904 e895, doi:10.1016/j.ccell.2018.03.017 (2018).

Hugo, W. et al. Genomic and Transcriptomic Features of Response to Anti-PD-1 Therapy in Metastatic Melanoma. Cell 165, 35-44, doi:10.1016/j.cell.2016.02.065 (2016). Bittner, M. et al. Molecular classification of cutaneous malignant melanoma by gene expression profiling. Nature 406, 536-540, doi:10.1038/35020115 (2000). Wellbrock, C. \& Arozarena, I. Microphthalmia-associated transcription factor in melanoma development and MAP-kinase pathway targeted therapy. Pigment Cell Melanoma Res 28, 390-406, doi:10.1111/pcmr.12370 (2015). 

cells. PLoS One 4, e8461, doi:10.1371/journal.pone.0008461 (2009).

26 Rambow, F. et al. New Functional Signatures for Understanding Melanoma Biology from Tumor Cell Lineage-Specific Analysis. Cell Rep 13, 840-853, doi:10.1016/j.celrep.2015.09.037 (2015). Cancer Genome Atlas, N. Genomic Classification of Cutaneous Melanoma. Cell 161, 16811696, doi:10.1016/j.cell.2015.05.044 (2015). Ennen, M. et al. MITF-High and MITF-Low Cells and a Novel Subpopulation Expressing Genes of Both Cell States Contribute to Intra- and Intertumoral Heterogeneity of Primary Melanoma. Clin Cancer Res 23, 7097-7107, doi:10.1158/1078-0432.CCR-17-0010 (2017). Eichhoff, O. M. et al. The immunohistochemistry of invasive and proliferative phenotype switching in melanoma: a case report. Melanoma research 20, 349-355, doi:10.1097/CMR.0b013e32833bd89e (2010). Haqq, C. et al. The gene expression signatures of melanoma progression. Proc Natl Acad Sci U S A 102, 6092-6097, doi:10.1073/pnas.0501564102 (2005).

31 Harbst, K. et al. Molecular and genetic diversity in the metastatic process of melanoma. The Journal of pathology 233, 39-50, doi:10.1002/path.4318 (2014).

Alexaki, V. I. et al. GLI2-mediated melanoma invasion and metastasis. Journal of the National Cancer Institute 102, 1148-1159, doi:10.1093/jnci/djq257 (2010). Pinner, S. et al. Intravital imaging reveals transient changes in pigment production and Brn2 expression during metastatic melanoma dissemination. Cancer research 69, 7969-7977, doi:10.1158/0008-5472.CAN-09-0781 (2009).

Dissanayake, S. K. et al. Wnt5A regulates expression of tumor-associated antigens in melanoma via changes in signal transducers and activators of transcription 3 phosphorylation. Cancer research 68, 10205-10214, doi:10.1158/0008-5472.CAN-08-2149 (2008).

O'Connell, M. P. et al. The orphan tyrosine kinase receptor, ROR2, mediates Wnt5A signaling in metastatic melanoma. Oncogene 29, 34-44, doi:10.1038/onc.2009.305 (2010). Weeraratna, A. T. et al. Wnt5a signaling directly affects cell motility and invasion of metastatic melanoma. Cancer Cell 1, 279-288 (2002). Bald, T. et al. Ultraviolet-radiation-induced inflammation promotes angiotropism and metastasis in melanoma. Nature 507, 109-113, doi:10.1038/nature13111 (2014). Riesenberg, S. et al. MITF and c-Jun antagonism interconnects melanoma dedifferentiation with pro-inflammatory cytokine responsiveness and myeloid cell recruitment. Nat Commun $\mathbf{6}$, 8755, doi:10.1038/ncomms9755 (2015). Carreira, S. et al. Mitf regulation of Dia1 controls melanoma proliferation and invasiveness. Genes Dev 20, 3426-3439, doi:10.1101/gad.406406 (2006).

40 Wellbrock, C. \& Marais, R. Elevated expression of MITF counteracts B-RAF-stimulated melanocyte and melanoma cell proliferation. The Journal of cell biology 170, 703-708, doi:10.1083/jcb.200505059 (2005).

41 Lauss, M. et al. Genome-Wide DNA Methylation Analysis in Melanoma Reveals the Importance of CpG Methylation in MITF Regulation. J Invest Dermatol 135, 1820-1828, doi:10.1038/jid.2015.61 (2015).

Cheng, P. F. et al. Methylation-dependent SOX9 expression mediates invasion in human melanoma cells and is a negative prognostic factor in advanced melanoma. Genome biology 16, 42, doi:10.1186/s13059-015-0594-4 (2015). Morrison, S. J., White, P. M., Zock, C. \& Anderson, D. J. Prospective identification, isolation by flow cytometry, and in vivo self-renewal of multipotent mammalian neural crest stem cells. Cell 96, 737-749 (1999). Cheli, Y. et al. CD271 is an imperfect marker for melanoma initiating cells. Oncotarget 5, 5272-5283, doi:10.18632/oncotarget.1967 (2014).

45 Held, M. A. et al. Characterization of melanoma cells capable of propagating tumors from a single cell. Cancer research 70, 388-397, doi:10.1158/0008-5472.CAN-09-2153 (2010). Quintana, E. et al. Phenotypic heterogeneity among tumorigenic melanoma cells from patients that is reversible and not hierarchically organized. Cancer Cell 18, 510-523, doi:10.1016/j.ccr.2010.10.012 (2010). Boiko, A. D. et al. Human melanoma-initiating cells express neural crest nerve growth factor receptor CD271. Nature 466, 133-137, doi:10.1038/nature09161 (2010). melanoma. Nat Commun 8, 1988, doi:10.1038/s41467-017-01573-6 (2017). 
49 Caramel, J. et al. A switch in the expression of embryonic EMT-inducers drives the development of malignant melanoma. Cancer Cell 24, 466-480, doi:10.1016/j.ccr.2013.08.018 (2013).

50 Wardwell-Ozgo, J. et al. HOXA1 drives melanoma tumor growth and metastasis and elicits an invasion gene expression signature that prognosticates clinical outcome. Oncogene 33, 10171026, doi:10.1038/onc.2013.30 (2014).

51 Ferretti, R., Bhutkar, A., McNamara, M. C. \& Lees, J. A. BMI1 induces an invasive signature in melanoma that promotes metastasis and chemoresistance. Genes Dev 30, 18-33, doi:10.1101/gad.267757.115 (2016).

Pastushenko, I. et al. Identification of the tumour transition states occurring during EMT. Nature 556, 463-468, doi:10.1038/s41586-018-0040-3 (2018).

53 Khoja, L. et al. Prevalence and heterogeneity of circulating tumour cells in metastatic cutaneous melanoma. Melanoma research 24, 40-46, doi:10.1097/CMR.0000000000000025 (2014).

$54 \mathrm{Yu}, \mathrm{M}$. et al. Circulating breast tumor cells exhibit dynamic changes in epithelial and mesenchymal composition. Science 339, 580-584, doi:10.1126/science.1228522 (2013). Tabassum, D. P. \& Polyak, K. Tumorigenesis: it takes a village. Nat Rev Cancer 15, 473-483, doi:10.1038/nrc3971 (2015).

56 Chapman, A. et al. Heterogeneous tumor subpopulations cooperate to drive invasion. Cell Rep 8, 688-695, doi:10.1016/j.celrep.2014.06.045 (2014).

57 Maurus, K. et al. The AP-1 transcription factor FOSL1 causes melanocyte reprogramming and transformation. Oncogene 36, 5110-5121, doi:10.1038/onc.2017.135 (2017).

58 Hong, X. et al. Molecular signatures of circulating melanoma cells for monitoring early response to immune checkpoint therapy. Proc Natl Acad Sci U S A 115, 2467-2472, doi:10.1073/pnas.1719264115 (2018).

59 Widmer, D. S. et al. Hypoxia contributes to melanoma heterogeneity by triggering HIF1alphadependent phenotype switching. J Invest Dermatol 133, 2436-2443, doi:10.1038/jid.2013.115 (2013).

60 Arozarena, I. \& Wellbrock, C. Targeting invasive properties of melanoma cells. The FEBS journal 284, 2148-2162, doi:10.1111/febs.14040 (2017).

61 Bertolotto, C. et al. A SUMOylation-defective MITF germline mutation predisposes to melanoma and renal carcinoma. Nature 480, 94-98, doi:10.1038/nature10539 (2011).

62 McGill, G. G., Haq, R., Nishimura, E. K. \& Fisher, D. E. c-Met expression is regulated by Mitf in the melanocyte lineage. The Journal of biological chemistry 281, 10365-10373, doi:10.1074/jbc.M513094200 (2006).

63 Hoek, K. S. et al. Novel MITF targets identified using a two-step DNA microarray strategy. Pigment Cell Melanoma Res 21, 665-676, doi:10.1111/j.1755-148X.2008.00505.x (2008).

64 Loftus, S. K. et al. Comparison of melanoblast expression patterns identifies distinct classes of genes. Pigment Cell Melanoma Res 22, 611-622, doi:10.1111/j.1755-148X.2009.00584.X (2009).

65 Bautista, N. C., Cohen, S. \& Anders, K. H. Benign melanocytic nevus cells in axillary lymph nodes. A prospective incidence and immunohistochemical study with literature review. American journal of clinical pathology 102, 102-108 (1994). Patterson, J. W. Nevus cell aggregates in lymph nodes. American journal of clinical pathology 121, 13-15, doi:10.1309/JXE9-EYQX-D691-LV2Y (2004). Chitty, J. L. et al. Recent advances in understanding the complexities of metastasis. F1000Research 7, doi:10.12688/f1000research.15064.2 (2018). Roesch, A. et al. Overcoming intrinsic multidrug resistance in melanoma by blocking the mitochondrial respiratory chain of slow-cycling JARID1B(high) cells. Cancer Cell 23, 811825, doi:10.1016/j.ccr.2013.05.003 (2013).

69 Wellbrock, C. et al. Oncogenic BRAF regulates melanoma proliferation through the lineage specific factor MITF. PLoS One 3, e2734, doi:10.1371/journal.pone.0002734 (2008).

70 Wellbrock, C., Weisser, C., Geissinger, E., Troppmair, J. \& Schartl, M. Activation of p59(Fyn) leads to melanocyte dedifferentiation by influencing MKP-1-regulated mitogenactivated protein kinase signaling. The Journal of biological chemistry 277, 6443-6454, doi:10.1074/jbc.M110684200 (2002).

71 Bogunovic, D. et al. Immune profile and mitotic index of metastatic melanoma lesions enhance clinical staging in predicting patient survival. Proc Natl Acad Sci U S A 106, 2042920434, doi:10.1073/pnas.0905139106 (2009). 

metastatic growth after dissemination of melanoma. Scientific reports 7, 10909, doi:10.1038/s41598-017-11366-y (2017). clinical outcome. Journal of the National Cancer Institute 98, 472-482, doi:10.1093/jnci/djj103 (2006).

74 Harbst, K. et al. Molecular profiling reveals low- and high-grade forms of primary melanoma. Clin Cancer Res 18, 4026-4036, doi:10.1158/1078-0432.CCR-12-0343 (2012). Jonsson, G. et al. Gene expression profiling-based identification of molecular subtypes in stage IV melanomas with different clinical outcome. Clin Cancer Res 16, 3356-3367, doi:10.1158/1078-0432.CCR-09-2509 (2010). Miskolczi, Z. et al. Collagen abundance controls melanoma phenotypes through lineagespecific microenvironment sensing. Oncogene 37, 3166-3182, doi:10.1038/s41388-018-02090 (2018).

77 Taylor, K. L. et al. Differentiated melanocyte cell division occurs in vivo and is promoted by mutations in Mitf. Development 138, 3579-3589, doi:10.1242/dev.064014 (2011). Kwa, M. Q., Herum, K. M. \& Brakebusch, C. Cancer-associated fibroblasts: how do they contribute to metastasis? Clinical \& experimental metastasis, doi:10.1007/s10585-019-099590 (2019).

Seip, K. et al. Fibroblast-induced switching to the mesenchymal-like phenotype and PI3K/mTOR signaling protects melanoma cells from BRAF inhibitors. Oncotarget 7, 1999720015, doi:10.18632/oncotarget.7671 (2016).

80 Kaur, A. et al. sFRP2 in the aged microenvironment drives melanoma metastasis and therapy resistance. Nature 532, 250-254, doi:10.1038/nature17392 (2016).

81 Kaur, A. et al. Remodeling of the Collagen Matrix in Aging Skin Promotes Melanoma Metastasis and Affects Immune Cell Motility. Cancer Discov 9, 64-81, doi:10.1158/21598290.CD-18-0193 (2019). photoprotection. Micron 35, 185-191, doi:10.1016/j.micron.2003.11.005 (2004). Smith, M. P. et al. The immune microenvironment confers resistance to MAPK pathway inhibitors through macrophage-derived TNFalpha. Cancer Discov 4, 1214-1229, doi:10.1158/2159-8290.CD-13-1007 (2014). Huergo-Zapico, L. et al. NK-cell Editing Mediates Epithelial-to-Mesenchymal Transition via Phenotypic and Proteomic Changes in Melanoma Cell Lines. Cancer research 78, 3913-3925, doi:10.1158/0008-5472.CAN-17-1891 (2018). Ohanna, M. et al. Senescent cells develop a PARP-1 and nuclear factor-\{kappa\}B-associated secretome (PNAS). Genes Dev 25, 1245-1261, doi:10.1101/gad.625811 (2011).

86 Wiedemann, G. M. et al. Microphthalmia-Associated Transcription Factor (MITF) Regulates Immune Cell Migration into Melanoma. Translational oncology 12, 350-360, doi:10.1016/j.tranon.2018.10.014 (2019). Vazquez, F. et al. PGC1alpha expression defines a subset of human melanoma tumors with increased mitochondrial capacity and resistance to oxidative stress. Cancer Cell 23, 287-301, doi:10.1016/j.ccr.2012.11.020 (2013). Hendrix, M. J. et al. Tumor cell vascular mimicry: Novel targeting opportunity in melanoma. Pharmacology \& therapeutics 159, 83-92, doi:10.1016/j.pharmthera.2016.01.006 (2016). Maniotis, A. J. et al. Vascular channel formation by human melanoma cells in vivo and in vitro: vasculogenic mimicry. The American journal of pathology 155, 739-752, doi:10.1016/S0002-9440(10)65173-5 (1999).

90 Mihic-Probst, D. et al. Tumor cell plasticity and angiogenesis in human melanomas. PLoS One 7, e33571, doi:10.1371/journal.pone.0033571 (2012).

91 Feige, E. et al. Hypoxia-induced transcriptional repression of the melanoma-associated oncogene MITF. Proc Natl Acad Sci U S A 108, E924-933, doi:10.1073/pnas.1106351108 (2011).

O'Connell, M. P. et al. Hypoxia induces phenotypic plasticity and therapy resistance in melanoma via the tyrosine kinase receptors ROR1 and ROR2. Cancer Discov 3, 1378-1393, doi:10.1158/2159-8290.CD-13-0005 (2013).

93 Nakazawa, M. S., Keith, B. \& Simon, M. C. Oxygen availability and metabolic adaptations. Nat Rev Cancer 16, 663-673, doi:10.1038/nrc.2016.84 (2016). 
94 Pascual, G. et al. Targeting metastasis-initiating cells through the fatty acid receptor CD36. Nature 541, 41-45, doi:10.1038/nature20791 (2017).

95 Silva, I. P. \& Long, G. V. Systemic therapy in advanced melanoma: integrating targeted therapy and immunotherapy into clinical practice. Current opinion in oncology 29, 484-492, doi:10.1097/CCO.0000000000000405 (2017).

96 Wellbrock, C. \& Arozarena, I. The Complexity of the ERK/MAP-Kinase Pathway and the Treatment of Melanoma Skin Cancer. Frontiers in cell and developmental biology 4, 33, doi:10.3389/fcell.2016.00033 (2016).

97 Arozarena, I. \& Wellbrock, C. Overcoming resistance to BRAF inhibitors. Annals of translational medicine 5, 387, doi:10.21037/atm.2017.06.09 (2017).

98 Ahmed, F. \& Haass, N. K. Microenvironment-Driven Dynamic Heterogeneity and Phenotypic Plasticity as a Mechanism of Melanoma Therapy Resistance. Frontiers in oncology 8, 173, doi:10.3389/fonc.2018.00173 (2018).

99 Larkin, J. et al. Combined Nivolumab and Ipilimumab or Monotherapy in Untreated Melanoma. The New England journal of medicine 373, 23-34, doi:10.1056/NEJMoa1504030 (2015).

100 Robert, C. et al. Pembrolizumab versus Ipilimumab in Advanced Melanoma. The New England journal of medicine 372, 2521-2532, doi:10.1056/NEJMoa1503093 (2015).

101 Schadendorf, D. et al. Pooled Analysis of Long-Term Survival Data From Phase II and Phase III Trials of Ipilimumab in Unresectable or Metastatic Melanoma. Journal of clinical oncology : official journal of the American Society of Clinical Oncology 33, 1889-1894, doi:10.1200/JCO.2014.56.2736 (2015).

102 Sharma, P., Hu-Lieskovan, S., Wargo, J. A. \& Ribas, A. Primary, Adaptive, and Acquired Resistance to Cancer Immunotherapy. Cell 168, 707-723, doi:10.1016/j.cell.2017.01.017 (2017).

103 Spranger, S., Bao, R. \& Gajewski, T. F. Melanoma-intrinsic beta-catenin signalling prevents anti-tumour immunity. Nature 523, 231-235, doi:10.1038/nature14404 (2015).

104 Zhu, J. et al. Resistance to cancer immunotherapy mediated by apoptosis of tumor-infiltrating lymphocytes. Nat Commun 8, 1404, doi:10.1038/s41467-017-00784-1 (2017).

105 Fallahi-Sichani, M. et al. Adaptive resistance of melanoma cells to RAF inhibition via reversible induction of a slowly dividing de-differentiated state. Mol Syst Biol 13, 905, doi:10.15252/msb.20166796 (2017).

106 Shaffer, S. M. et al. Rare cell variability and drug-induced reprogramming as a mode of cancer drug resistance. Nature 546, 431-435, doi:10.1038/nature22794 (2017).

$107 \mathrm{Su}, \mathrm{Y}$. et al. Single-cell analysis resolves the cell state transition and signaling dynamics associated with melanoma drug-induced resistance. Proc Natl Acad Sci U S A 114, 1367913684, doi:10.1073/pnas.1712064115 (2017).

108 Smith, M. P. et al. Inhibiting Drivers of Non-mutational Drug Tolerance Is a Salvage Strategy for Targeted Melanoma Therapy. Cancer Cell 29, 270-284, doi:10.1016/j.ccell.2016.02.003 (2016).

109 Smith, M. P. et al. Effect of SMURF2 targeting on susceptibility to MEK inhibitors in melanoma. Journal of the National Cancer Institute 105, 33-46, doi:10.1093/jnci/djs471 (2013).

110 Nazarian, R. et al. Melanomas acquire resistance to B-RAF(V600E) inhibition by RTK or NRAS upregulation. Nature 468, 973-977, doi:10.1038/nature09626

nature09626 [pii] (2010).

111 Villanueva, J. et al. Acquired resistance to BRAF inhibitors mediated by a RAF kinase switch in melanoma can be overcome by cotargeting MEK and IGF-1R/PI3K. Cancer Cell 18, 683695, doi:10.1016/j.ccr.2010.11.023 (2010).

112 Sun, C. et al. Reversible and adaptive resistance to BRAF(V600E) inhibition in melanoma. Nature 508, 118-122, doi:10.1038/nature13121 (2014).

113 Konieczkowski, D. J. et al. A melanoma cell state distinction influences sensitivity to MAPK pathway inhibitors. Cancer Discov 4, 816-827, doi:10.1158/2159-8290.CD-13-0424 (2014).

114 Muller, J. et al. Low MITF/AXL ratio predicts early resistance to multiple targeted drugs in melanoma. Nat Commun 5, 5712, doi:10.1038/ncomms6712 (2014).

115 Boshuizen, J. et al. Cooperative targeting of melanoma heterogeneity with an AXL antibodydrug conjugate and BRAF/MEK inhibitors. Nature medicine 24, 203-212, doi:10.1038/nm.4472 (2018). 
117 Yan, Y. et al. Genomic Features of Exceptional Response in Vemurafenib +/- Cobimetinibtreated Patients with BRAF (V600)-mutated Metastatic Melanoma. Clin Cancer Res, doi:10.1158/1078-0432.CCR-18-0720 (2019).

118 Menon, D. R. et al. A stress-induced early innate response causes multidrug tolerance in melanoma. Oncogene, doi:10.1038/onc.2014.432 (2015).

119 Sharma, S. V. et al. A chromatin-mediated reversible drug-tolerant state in cancer cell subpopulations. Cell 141, 69-80, doi:10.1016/j.cell.2010.02.027 (2010).

120 Smith, M. P. \& Wellbrock, C. Molecular Pathways: Maintaining MAPK Inhibitor Sensitivity by Targeting Nonmutational Tolerance. Clin Cancer Res 22, 5966-5970, doi:10.1158/10780432.CCR-16-0954 (2016).

121 Hugo, W. et al. Non-genomic and Immune Evolution of Melanoma Acquiring MAPKi Resistance. Cell 162, 1271-1285, doi:10.1016/j.cell.2015.07.061 (2015).

122 Kong, X. et al. Cancer drug addiction is relayed by an ERK2-dependent phenotype switch. Nature 550, 270-274, doi:10.1038/nature24037 (2017).

123 Obenauf, A. C. et al. Therapy-induced tumour secretomes promote resistance and tumour progression. Nature 520, 368-372, doi:10.1038/nature14336 (2015).

124 Smith, M. P. et al. Targeting endothelin receptor signalling overcomes heterogeneity driven therapy failure. EMBO Mol Med 9, 1011-1029, doi:10.15252/emmm.201607156 (2017).

125 Jones, P. A., Issa, J. P. \& Baylin, S. Targeting the cancer epigenome for therapy. Nature reviews. Genetics 17, 630-641, doi:10.1038/nrg.2016.93 (2016).

126 Rabbie, R., Ferguson, P., Molina-Aguilar, C., Adams, D. J. \& Robles-Espinoza, C. D. Melanoma subtypes: genomic profiles, prognostic molecular markers and therapeutic possibilities. The Journal of pathology 247, 539-551, doi:10.1002/path.5213 (2019).

127 Shain, A. H. et al. Exome sequencing of desmoplastic melanoma identifies recurrent NFKBIE promoter mutations and diverse activating mutations in the MAPK pathway. Nat Genet $\mathbf{4 7}$, 1194-1199, doi:10.1038/ng.3382 (2015).

128 Eroglu, Z. et al. High response rate to PD-1 blockade in desmoplastic melanomas. Nature 553, 347-350, doi:10.1038/nature25187 (2018).

129 Das Thakur, M. et al. Modelling vemurafenib resistance in melanoma reveals a strategy to forestall drug resistance. Nature 494, 251-255, doi:10.1038/nature11814 (2013).

130 Valpione, S. et al. Rechallenge with BRAF-directed treatment in metastatic melanoma: A multi-institutional retrospective study. European journal of cancer 91, 116-124, doi:10.1016/j.ejca.2017.12.007 (2018).

131 Vinal, D., Martinez, D. \& Espinosa, E. Efficacy of rechallenge with BRAF inhibition therapy in patients with advanced BRAFV600 mutant melanoma. Clinical \& translational oncology : official publication of the Federation of Spanish Oncology Societies and of the National Cancer Institute of Mexico, doi:10.1007/s12094-018-02028-0 (2019).

132 Smith, M. P. et al. A PAX3/BRN2 rheostat controls the dynamics of BRAF mediated MITF regulation in MITF(high) /AXL(low) melanoma. Pigment Cell Melanoma Res, doi:10.1111/pcmr.12741 (2018).

133 Haq, R. et al. Oncogenic BRAF regulates oxidative metabolism via PGC1alpha and MITF. Cancer Cell 23, 302-315, doi:10.1016/j.ccr.2013.02.003 (2013).

134 Haq, R. et al. BCL2A1 is a lineage-specific antiapoptotic melanoma oncogene that confers resistance to BRAF inhibition. Proc Natl Acad Sci U S A 110, 4321-4326, doi:10.1073/pnas.1205575110 (2013).

135 Luke, J. J., Flaherty, K. T., Ribas, A. \& Long, G. V. Targeted agents and immunotherapies: optimizing outcomes in melanoma. Nature reviews. Clinical oncology 14, 463-482, doi:10.1038/nrclinonc.2017.43 (2017).

136 Boni, A. et al. Selective BRAFV600E inhibition enhances T-cell recognition of melanoma without affecting lymphocyte function. Cancer research 70, 5213-5219, doi:10.1158/00085472.CAN-10-0118 (2010).

137 Zhao, F. et al. Paracrine Wnt5a-beta-Catenin Signaling Triggers a Metabolic Program that Drives Dendritic Cell Tolerization. Immunity 48, 147-160 e147, doi:10.1016/j.immuni.2017.12.004 (2018).

138 Greaves, M. \& Maley, C. C. Clonal evolution in cancer. Nature 481, 306-313, doi:10.1038/nature10762 (2012). 
139 Brinckerhoff, C. E. Cancer Stem Cells (CSCs) in melanoma: There's smoke, but is there fire? Journal of cellular physiology 232, 2674-2678, doi:10.1002/jcp.25796 (2017).

140 Perego, M. et al. Heterogeneous phenotype of human melanoma cells with in vitro and in vivo features of tumor-initiating cells. J Invest Dermatol 130, 1877-1886, doi:10.1038/jid.2010.69 (2010).

141 Roesch, A. et al. A temporarily distinct subpopulation of slow-cycling melanoma cells is required for continuous tumor growth. Cell 141, 583-594, doi:10.1016/j.cell.2010.04.020 (2010).

142 Spinella, F. et al. Endothelin-1 and endothelin-3 promote invasive behavior via hypoxiainducible factor-1alpha in human melanoma cells. Cancer research 67, 1725-1734, doi:10.1158/0008-5472.CAN-06-2606 (2007).

143 Cheli, Y. et al. Mitf is the key molecular switch between mouse or human melanoma initiating cells and their differentiated progeny. Oncogene 30, 2307-2318, doi:10.1038/onc.2010.598 (2011).

144 Nishimura, E. K. et al. Key roles for transforming growth factor beta in melanocyte stem cell maintenance. Cell stem cell 6, 130-140, doi:10.1016/j.stem.2009.12.010 (2010).

145 Webster, M. R. et al. Wnt5A promotes an adaptive, senescent-like stress response, while continuing to drive invasion in melanoma cells. Pigment Cell Melanoma Res 28, 184-195, doi:10.1111/pcmr.12330 (2015).

146 Richard, G. et al. ZEB1-mediated melanoma cell plasticity enhances resistance to MAPK inhibitors. EMBO Mol Med 8, 1143-1161, doi:10.15252/emmm.201505971 (2016).

147 Ramsdale, R. et al. The transcription cofactor c-JUN mediates phenotype switching and BRAF inhibitor resistance in melanoma. Science signaling $\mathbf{8}$, ra82, doi:10.1126/scisignal.aab1111 (2015).

148 Titz, B. et al. JUN dependency in distinct early and late BRAF inhibition adaptation states of melanoma. Cell discovery 2, 16028, doi:10.1038/celldisc.2016.28 (2016).

\section{Acknowledgements}

C.W acknowledges support by Cancer Research UK (CRUK) (grant number C11591/A16416). IA is supported by a Miguel Servet contract CP15/00176 from the "Instituto de Salud Carlos III-FEDER". We apologize to all colleagues whose work could not be cited here due to space limitations.

\section{Author contributions}

Both authors contributed equally to writing the article.

\section{Competing interests}

The authors to declare no competing interests in relation to the work described. 
Fig 1
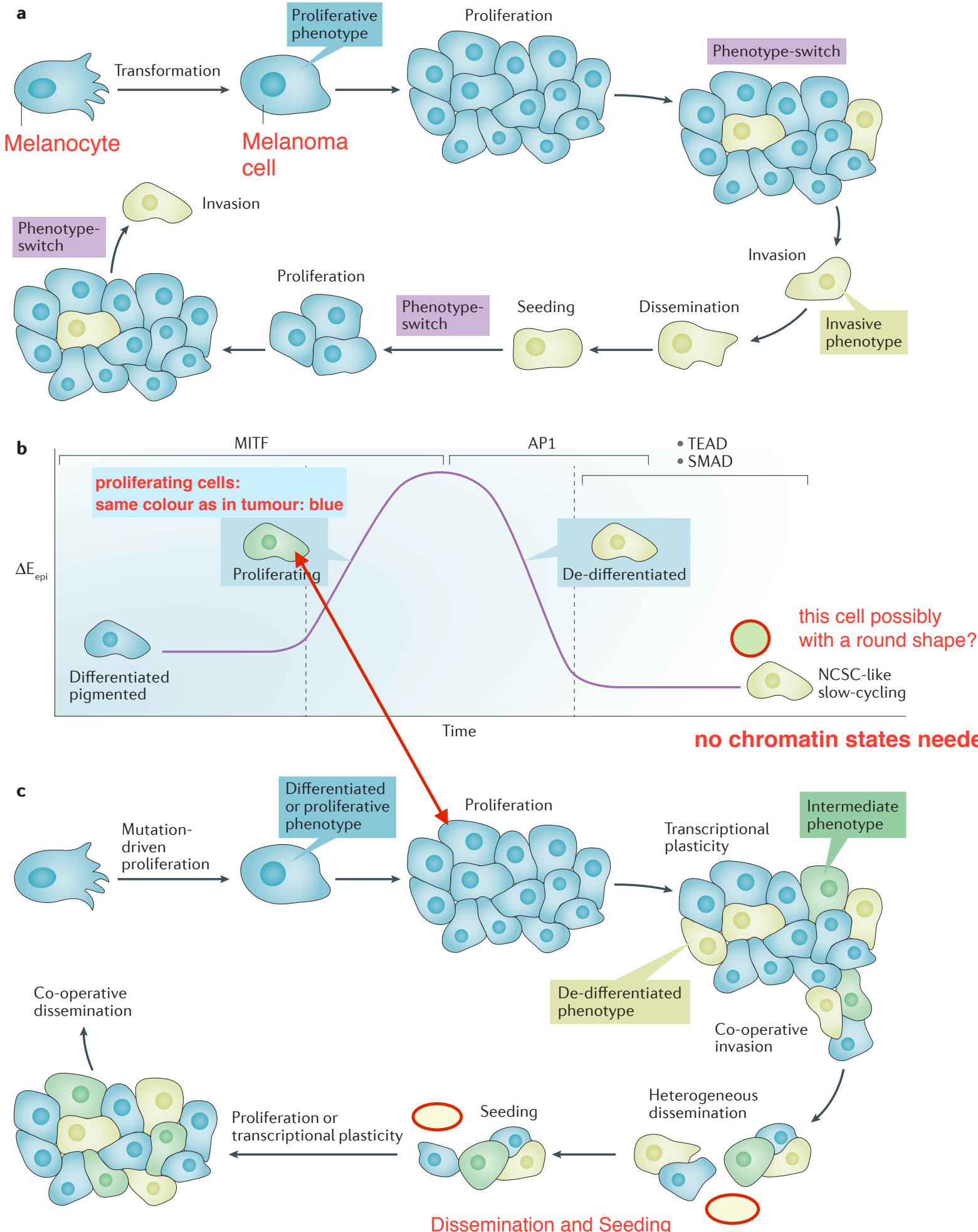
can also be performed by single cells. This needs to Nature Reviews | Cancer be indicated. 
Fig 2

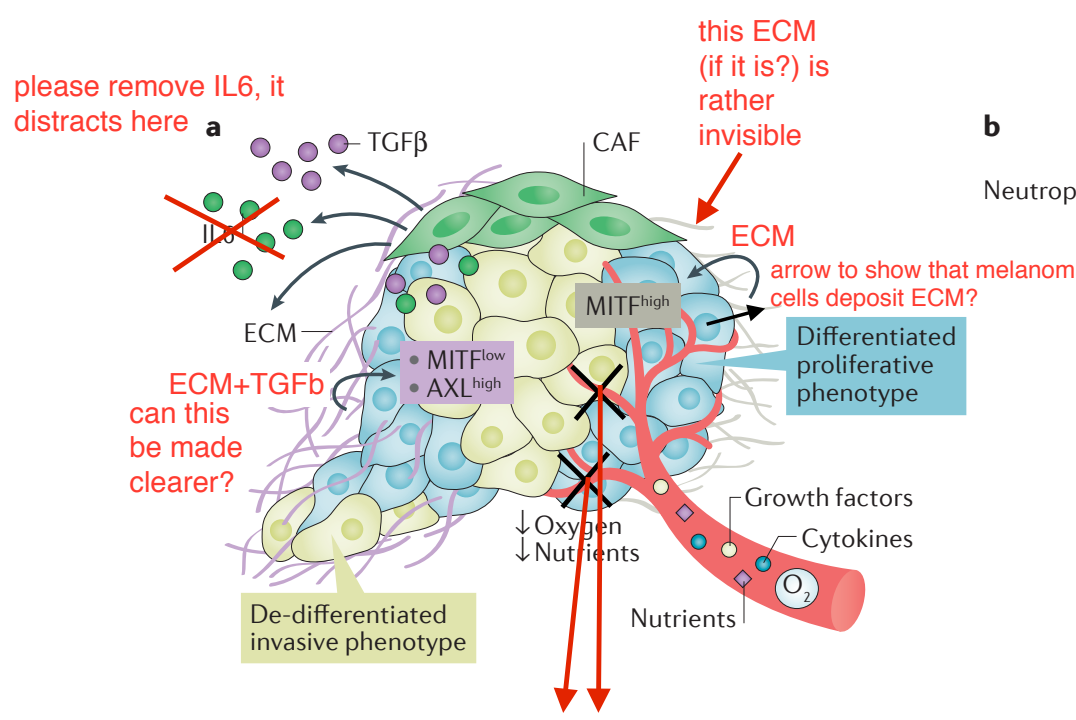

please NO connection of vasculature to light green, dedifferentiated cells. The absence of vasculature is the reason for oxygen and nutrients down, which induces light green cells. please remove UV: UV acts on keratinocytes, which release HMGB1, which recruits

neutrophils

so it's rather indirect TNF is also produced

by lymphocytes and myeloid cells under different circumstances

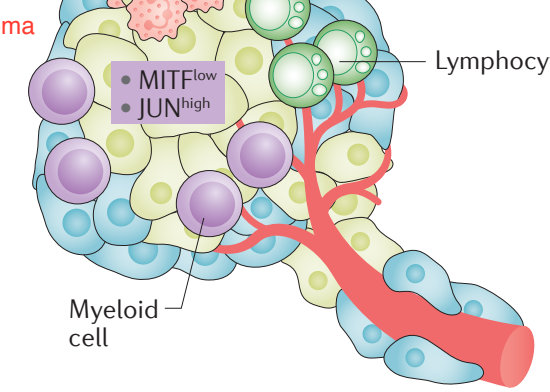

Nature Reviews | Cancer 
Fig 3 I have amended these figures and they are included below I have adjusted the figure legends accordingly

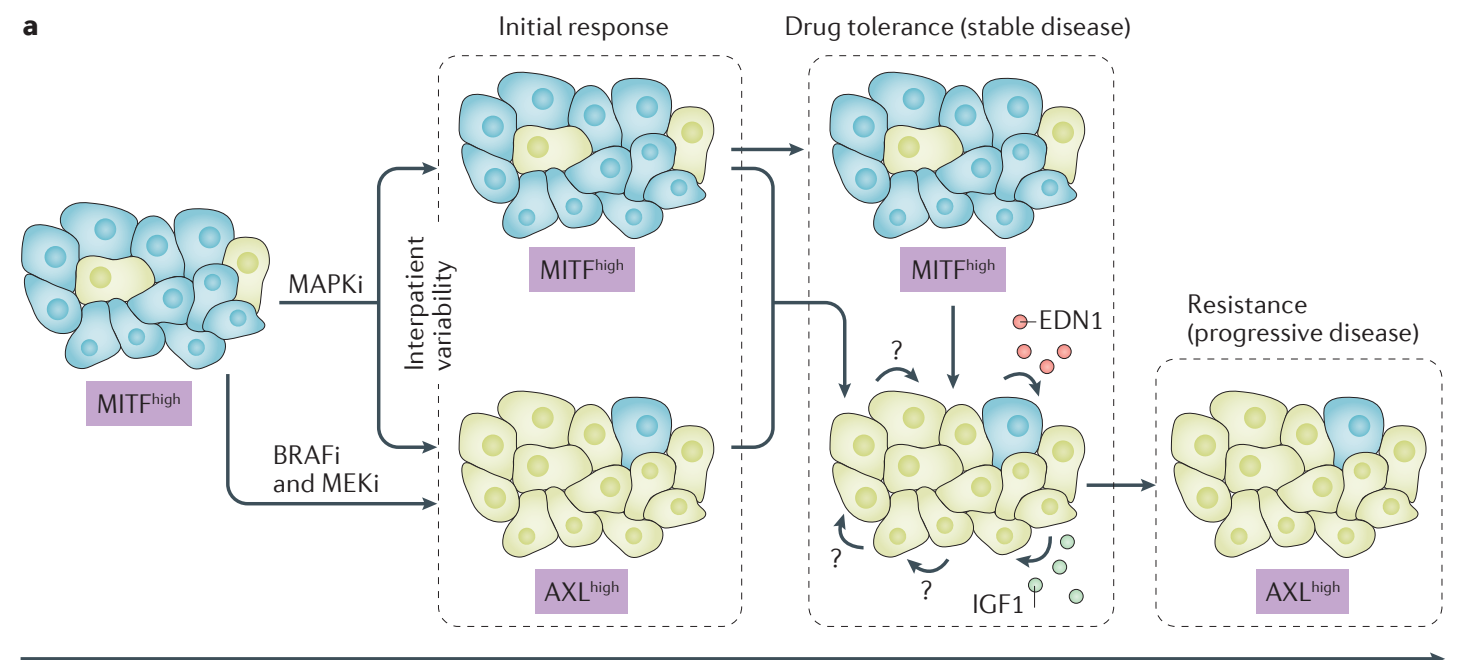

Ongoing treatment
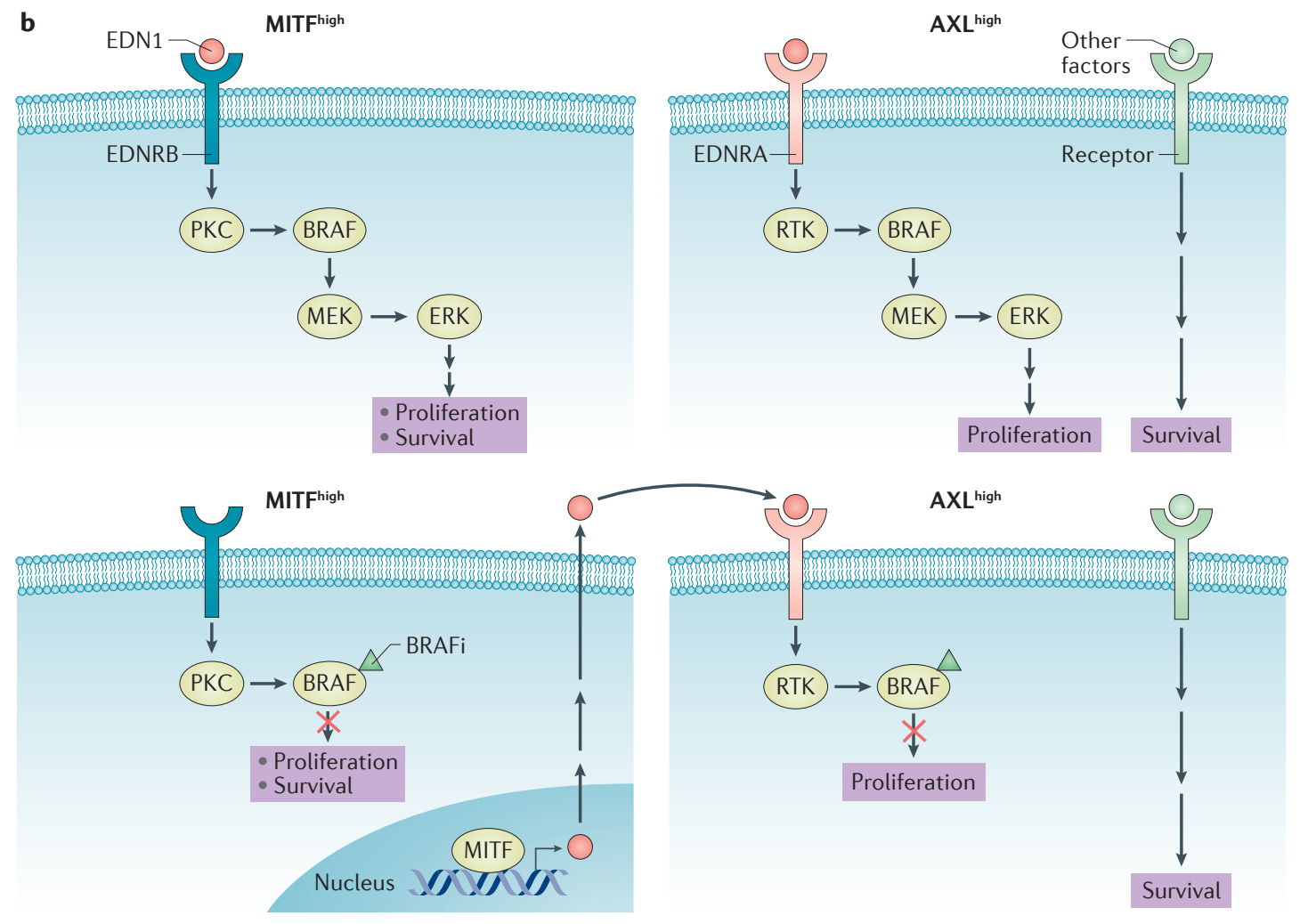


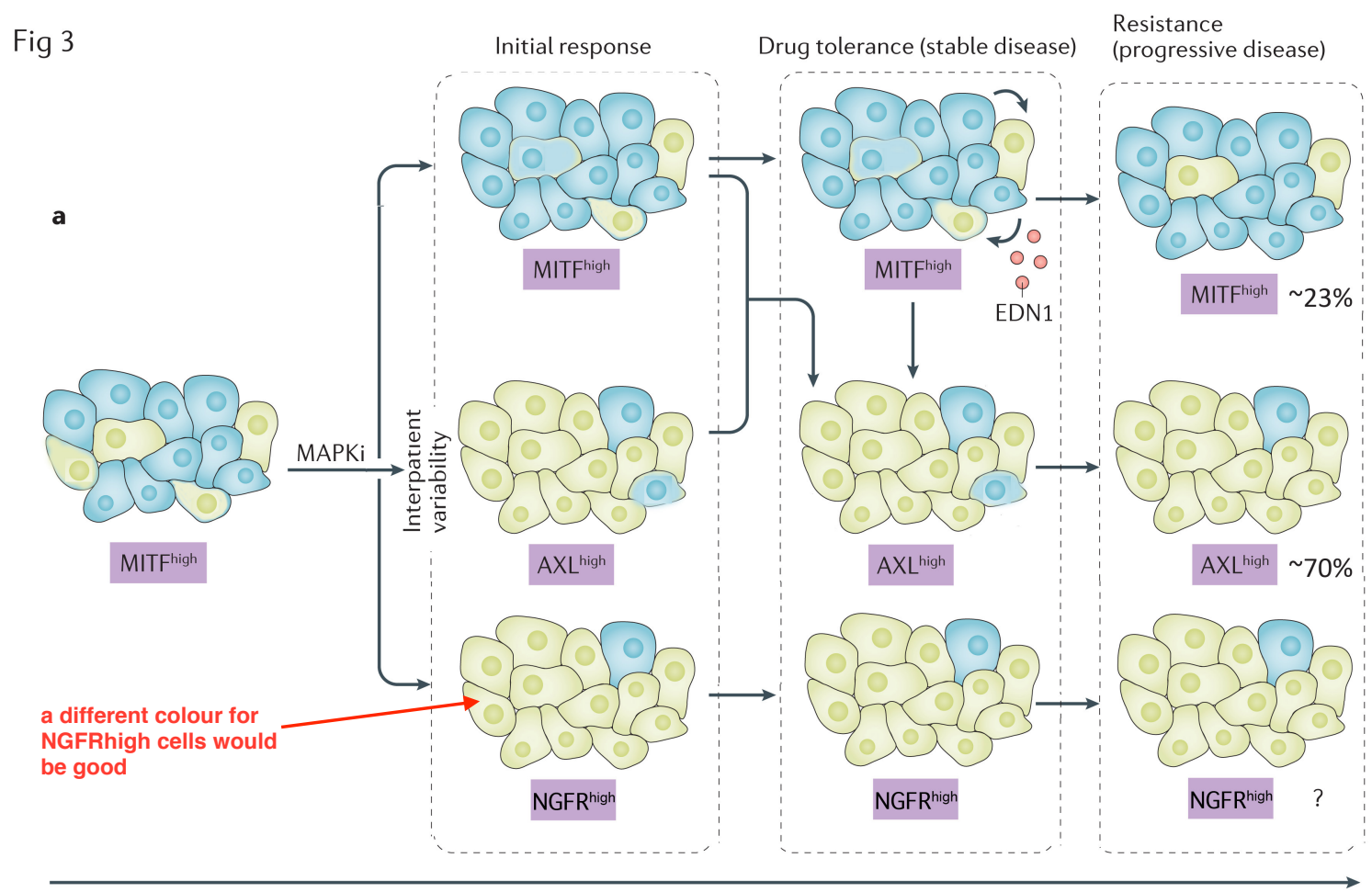

Ongoing treatment 

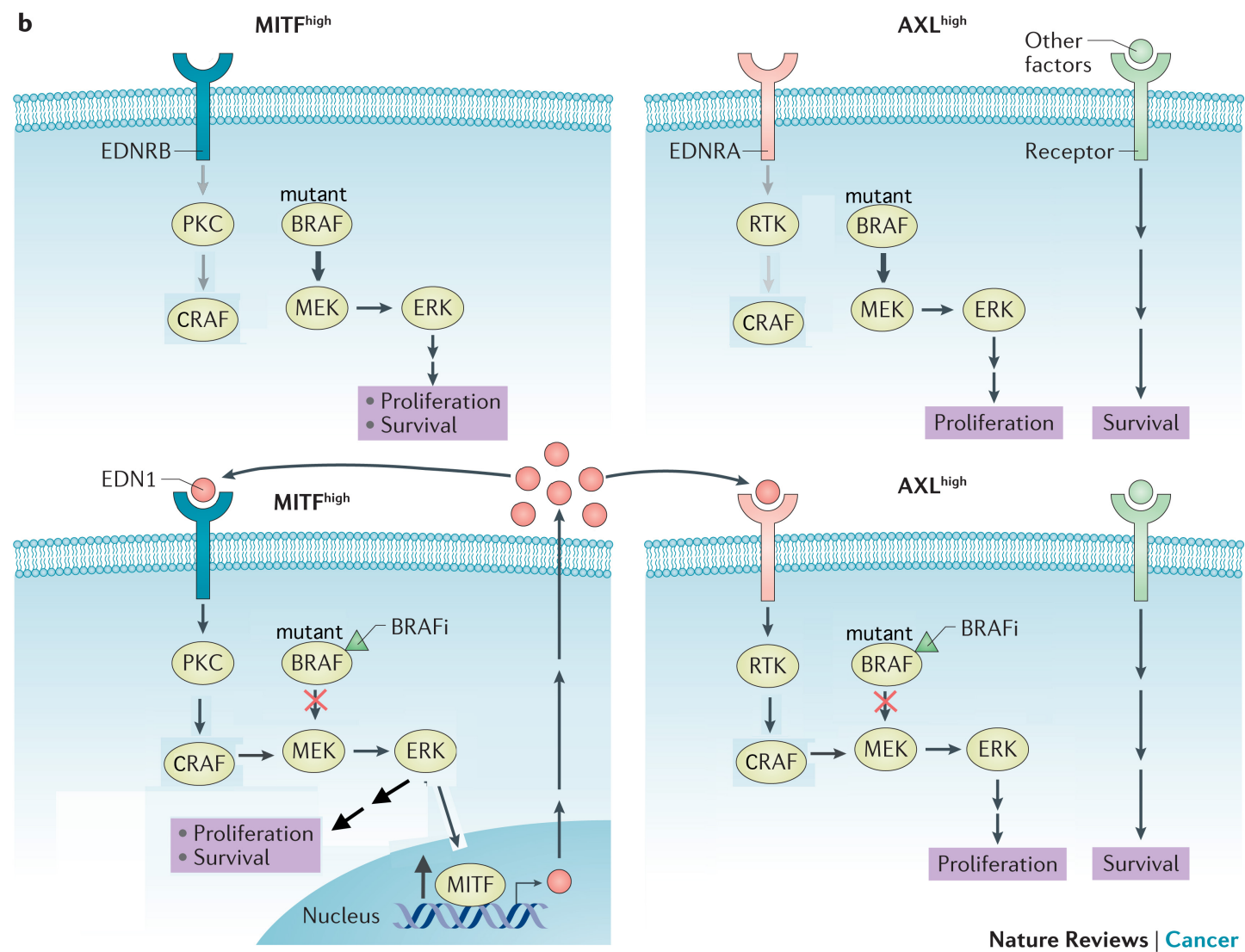

Nature Reviews | Cancer 